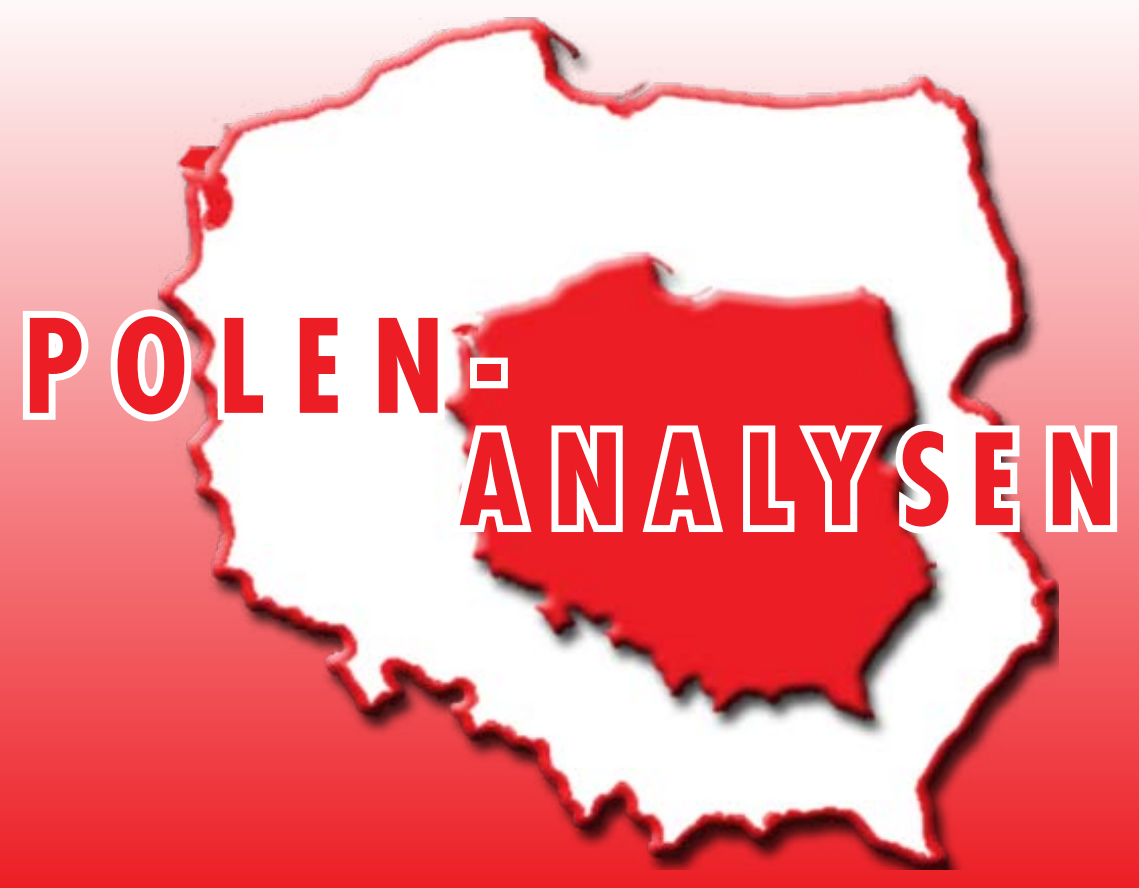

www.laender-analysen.de/polen

\title{
KOHABITATION IN POLEN
}

ANALYSE

Kohabitation nowy

Artur Kopka, Frankfurt (Oder), und Claudia Schäfer, Freiburg

- TABELLEN UND GRAFIKEN

Umfragen zur Kohabitation in Polen nach 2007

CHRONIK

Vom 03. bis zum 16. November 2009

VERANSTALTUNGSHINWEIS

Call for Applications $-5^{\text {th }}$ Changing Europe Summer School 


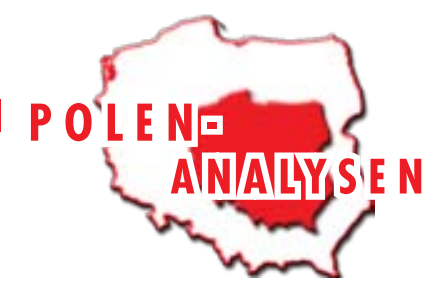

Analyse

\section{Kohabitation nowy}

Artur Kopka, Frankfurt (Oder), und Claudia Schäfer, Freiburg

\section{Zusammenfassung}

Probleme der Kohabitation von PiS-Staatspräsident Lech Kaczyński und PO-Ministerpräsident Donald Tusk, die aufgrund der verfassungsrechtlichen Bestimmungen in Kernbereichen der Innen- und Außenpolitik auftreten und die Effektivität des Regierungshandelns beeinträchtigen, sind Gegenstand der Analyse. Nach einer kurzen Erläuterung der verfassungsrechtlichen Entwicklung werden die Konfliktsituationen in der Außenund Innenpolitik zwischen den beiden Institutionen der doppelköpfigen Exekutive skizziert, um anschließend die gesellschaftliche Wahrnehmung des Konflikts zu beleuchten.

"K to rządzi Polską?«, "Wer regiert Polen?« fragte im August 2008 Newsweek Polska und brachte damit eine aktuelle Problemlage der polnischen Politik auf den Punkt, die spätestens seit den Differenzen um die Repräsentation Polens bei den europäischen Gipfeln auch ein europaweites Medienecho hervorruft.

Schon als Jarosław Kaczyński Minuten nach der Verkündung des Wahlergebnisses der Parlamentswahl vom 21. Oktober 2007 eine "entschiedene und harte Opposition" ankündigte, drängte sich der Schluss auf, dass er diese nicht zuletzt durch seinen Zwillingsbruder Lech zu betreiben gedachte. Glaubte man dagegen den Aussagen des Staatspräsidenten Lech Kaczyński selbst, dessen gemäßigteres politisches Profil als positive Ausgangslage gedeutet werden konnte und der in einem Interview mit der Tageszeitung Rzeczpospolita zehn Tage später betonte, dass er nur selten von seinem Vetorecht Gebrauch machen und sich ganz klar auf seine verfassungsmäßigen Rechte beschränken würde, könnte diese neue Spielart des Nebeneinander von Regierungschef und Staatspräsident aus verschiedenen politischen Lagern - Kohabitation genannt - tatsächlich günstigere Bedingungen vorfinden als die früheren Kohabitationen unter der »Kleinen Verfassung « (KV) von 1992 und der »Neuen Verfassung« (NV) von 1997.

Mit dem vorläufigen Ende der »IV. Republik« durch die Ablösung von Ministerpräsident Jarosław Kaczyński (Recht und Gerechtigkeit / Prawo i Sprawiedliwość-PiS) durch Donald Tusk (Bürgerplattform / Platforma Obywatelska - PO) infolge der Parlamentswahl war in Warschau ein exekutives Nebeneinander von zwei Lagern des Mitte-Rechts-Spektrums eingetreten, die sich nicht so sehr in der Programmatik wie im politischen Stil unterscheiden. Auch wenn die Debatte um den außenpolitischen Kurs Tusks sowie die für Polen so wichtige Frage nach der eigenen Rolle in Europa regelmäßig wesentlich ausführlicher thematisiert wird als die institutionelle Entwicklung, stellt sich die Frage nach dem Zusammen- wirken von Donald Tusk und Präsident Lech Kaczyński (PiS) ganz grundsätzlich, denn die neue Konstellation mit Donald Tusk als Ministerpräsidenten und Staatspräsident Lech Kaczyński, dessen Amtszeit noch bis mindestens 2010 andauert, weicht insofern von der bisherigen Kohabitations-Situation zwischen postkommunistischen und Post-Solidarność-Politikern ab, als beide Parteien und Politiker aus einem in sich zerstrittenen Lager stammen.

Welche Kompetenzkonflikte sind seit 2007 aufgetreten? Wie sind deren Auswirkungen auf die politische Stabilität in Polen zu bewerten? Welche Unterschiede sind gegenüber den früheren Phasen der Kohabitation zu konstatieren? Diese Fragen sollen im Folgenden näher beleuchtet werden - erstens durch eine Annäherung an die verfassungsrechtlichen Grundlagen des Verhältnisses von Präsident und Ministerpräsident in der Neuen Verfassung von 1997, zweitens durch die Analyse der neuen politischen Situation seit 2007 und drittens durch ein kurzes Schlaglicht auf die gesellschaftliche Wahrnehmung des Konflikts.

\section{Verfassungsrechtliche Grundlagen des Konflikts}

Die in der NV eindeutiger definierten Rollen weisen Parlament und Regierung, nicht dem Präsidenten, eine "dominante Position innerhalb der Staatsleitung " (Art. 146 NV) zu. Dabei liegen einige ehemalige Präsidialkompetenzen nun ausdrücklich in der Hand des Ministerrates, etwa die allgemeine Leitung im Bereich der Beziehungen zu anderen Staaten und internationalen Organisationen (Art. 52 NV). Im Vergleich zur KV werden die Kompetenzen des Präsidenten eingeschränkt (Art. 126 III NV), denn das Gesetzesveto des Präsidenten kann nun bereits mit einer 3/5-Mehrheit zurückgewiesen werden (Art. 122 NV), er kann kein Veto mehr gegen den Haushalt einlegen und er muss sich für ein Veto oder die Weiterleitung an das Verfassungsgericht 


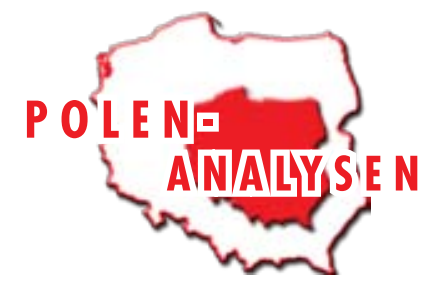

entscheiden, da eine Weiterleitung an das höchste Judikativorgan nicht mehr möglich ist, wenn das Parlament das Veto bereits erfolgreich abgewehrt hat. Insbesondere bedarf der Wille des Präsidenten der Vermittlung durch die Regierung, indem der Ministerpräsident alle nicht in den Bereich ausdrücklich aufgelisteter Machtkompetenzen fallenden Amtspapiere des Präsidenten gegenzeichnen muss. Damit erstarkte die "systemgebundene Stellung der Regierung « und insbesondere des Ministerpräsidenten.

Trotz der hier nachgezeichneten Veränderungen im Detail ist kritisiert worden, dass die NV keine Vorschläge enthalte, die tieferliegenden Ursachen für die immer wiederkehrenden institutionellen Spannungen der Zeit von 1989 bis 1997 zu beheben. Trotz positiver Konsolidierungsfortschritte ist die Diskussion um staatliche Institutionen nicht beendet. Seit dem Wahlsieg der PiS im Herbst 2005 und der Wahl Lech Kaczyńskis zum Präsidenten zielte die Agenda von der »IV. Republik» u. a. auch auf den Umbau der staatlichen Institutionen zugunsten eines starken Präsidenten, der endlich Stabilität in die nach wie vor wechselhafte politische (Parteien-)Landschaft bringen sollte. Dabei wird konsequent übersehen, dass es gerade der balancierte Semi-Präsidentialismus (bzw. das parlamentarisch-präsidentielle System) der Verfassung von 1997 war, der mit immerhin vier Jahren Regierung Buzek und zweieinhalb Jahren Regierung Miller mehr Stabilität und Effizienz hervorbrachte. Mit dem Untergang des Projekts der »IV. Republik« zeichnete sich allerdings auch keine verfassungsändernde Mehrheit mehr ab, die eine Stärkung der Macht des Präsidenten zulasten des Parlamentes tatsächlich hätte umsetzen können.

\section{Lech Kaczyński und die neue politische Situation nach 2007}

Bereits unmittelbar nach den Wahlen von 2007 deutete die längere Abwesenheit von Präsident Lech Kaczyński in der Öffentlichkeit darauf hin, dass dieser sich mit der neuen politischen Situation nur schwer würde arrangieren können. Auch mit der absichtlich verzögerten Nominierung von Donald Tusk zum Ministerpräsidenten und dem mehr oder weniger direkten Versuch, auf die Besetzung bestimmter Ministerien Einfluss zu nehmen, bewies er die emotionale Bindung an die Partei seines Bruders und dass er sich nicht als unparteiischer Präsident, sondern vielmehr als Vertreter der PiS-Programmatik verstehen würde. Es wurde klar, dass das Staatsoberhaupt nach Übernahme der Regierungsverantwortung durch die Koalition der PO mit der Polnischen Bauernpartei (Polskie Stronnictwo Ludowe - PSL) zum Haupt- akteur der Opposition werden würde. Einen geradezu symbolischen Charakter hatten dabei die beiden Feierlichkeiten zur Übernahme der Regierungsverantwortung. Zunächst bedankte sich der Präsident während einer groß angelegten Feier mit einer Festrede im Säulensaal des Präsidentenpalais bei seinem Bruder Jarosław Kaczyński für dessen Regierungszeit, wogegen die Vereidigung von Donald Tusk zum neuen Ministerpräsidenten nicht einmal 10 Minuten dauerte und im kleinen Nebensaal abgehalten wurde.

Dass der Präsident sich gerade die Außenpolitik für die Arena seiner Auseinandersetzungen mit der Regierung aussuchen würde, also den Bereich, wo die Auslegung der verfassungsrechtlichen Formulierungen hinsichtlich der Kompetenzen zwischen den beiden exekutiven Machtzentren einen gewissen Interpretationsspielraum zulässt und daher bestimmte Konfliktpotenziale in sich trägt, war abzusehen. Durch die Nominierung der ehemaligen Außenministerin in der Regierung Kaczyński, Anna Fotyga, zur Beraterin des Präsidenten und später zur Leiterin der Präsidialkanzlei war ein Konflikt in der Außenpolitik vorprogrammiert, da Fotyga weniger auf Zusammenarbeit als vielmehr auf Kritik an der Regierungspolitik ausgerichtet war. Die gravierenden außenpolitischen Unterschiede zwischen Präsident und Regierung wurden bald deutlich. Zu den drei außenpolitischen Hauptbereichen, die bisher durch starke Differenzen zwischen Tusk und Kaczyński gekennzeichnet waren, gehören das Verhältnis zu den USA, zu Russland und zur EU. Es zeigten sich dabei zwei unterschiedliche Visionen von Außenpolitik, die pro-europäische von Tusk, die eine engere Zusammenarbeit mit den wichtigsten Ländern der EU in den Mittelpunkt stellt, und die stark pro-amerikanische und anti-russische von Kaczyński, die vor allem durch die angestrebte Integration der Ukraine, Georgiens und anderer post-sowjetischer Staaten in den Westen gekennzeichnet ist. Dabei stützt Lech Kaczyński seine Argumente hauptsächlich auf eine bei der PiS-Wählerschaft bewährte Rhetorik, die vor allem auf "polnischer Würde«, Misstrauen gegenüber der EU, Skepsis bezüglich der europäischen Integration, antideutschen Stimmungen und einer Politik gegenüber Russland, die nicht »auf Knien« geführt werden solle, aufbaut.

\section{Konflikte in der außenpolitischen Arena}

Die ersten Spannungen in der Außenpolitik ließen nicht lange auf sich warten. Bereits im Dezember 2007 kündigte die Regierung an, auch gegen den Willen des Präsidenten das Truppenkontingent aus dem Irak zurückzuziehen. Kurz danach folgte der Konflikt um die Vertre- 


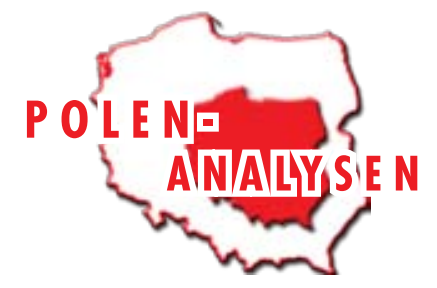

tung polnischer Interessen bei den zwei wichtigen EUGipfeltreffen in Lissabon und Brüssel, obwohl sich in der polnischen Außenpolitik bereits etabliert hatte, dass Polen bei den EU-Gipfeln, bei denen vorwiegend wirtschaftliche Angelegenheiten thematisiert werden, vom Regierungschef und bei den NATO-Treffen, wo es um Sicherheitsfragen geht, vom Präsidenten als dem obersten Befehlshaber der Streitkräfte repräsentiert wird. In diesem Falle wurde versucht, eine Auseinandersetzung zwischen den beiden Staatsorganen durch einen Kompromiss zu vermeiden, nach dem in Lissabon Präsident Kaczyński als Chef der polnischen Delegation fungieren, dann aber in Brüssel dem Ministerpräsidenten den ersten Platz einräumen sollte. Unverständlich und verblüffend wirkte dabei insbesondere das Verhalten Lech Kaczyńskis, als er im März 2008 in den Medien die Kritik der PiS am Lissabon-Vertrag unterstützte, obwohl dieser Vertrag doch von ihm persönlich ausgehandelt worden war. Erst nach einem »Kompromiss mit der Regierung« erklärte er sich bereit, den Vertrag zu ratifizieren, unterschrieb ihn jedoch erst geraume Zeit später nach dem Referendum in Irland, nämlich am 10. Oktober 2009.

$\mathrm{Zu}$ einer deutlichen Konfliktverschärfung kam es im Juni 2008 wegen der Verhandlungen mit den USA über die Installation von Teilen eines amerikanischen Raketenabwehrsystems in Polen, das in dem ursprünglichen Umfang von der Regierung Obama nicht mehr weiterverfolgt wird. Der Erfolg dieses Projekts war dem Präsidenten sehr wichtig, weil er ihn als Priorität seiner Außenpolitik verstand. Die Regierung wollte aber für die Stationierung der Raketenabwehr auf polnischem Gebiet von den Amerikanern zusätzliche Zugeständnisse zugunsten der polnische Streitkräfte aushandeln. Nach einem Gerücht, dass die Regierung die Gespräche abzubrechen drohe, schickte der Präsident seine Ministerin Anna Fotyga zu klärenden Gesprächen in die USA, um dafür zu sorgen, dass der mächtige Verbündete in keinem Fall »beleidigt « würde. Dieses Verhalten schwächte die Verhandlungsposition der Regierung erheblich. Kurz danach lud Lech Kaczyński Außenminister Sikorski in den Präsidentenpalast und befragte diesen intensiv zu diesem Thema in einem schalldichten Raum, was als ein weiteres Zeichen eines starken Misstrauens gegenüber der Regierungspolitik interpretiert wurde. Darüber hinaus gelangten dabei einige Aufnahmen aus diesem angeblich geheimen Gespräch an die Öffentlichkeit, was zu einer spektakulären Blamage für die Präsidialkanzlei führte.

Im Laufe der Georgien-Krise kritisierte Donald Tusk den Präsidenten für die seiner Meinung nach »zu starken
Worte«, die Lech Kaczyński bei seiner engagierten Rede in Tiflis gegenüber Russland gewählt hatte. Obwohl die Reise nach Georgien zu den spektakulärsten außenpolitischen Erfolgen von Lech Kaczyński gezählt werden kann, machte sich auch bei dieser Gelegenheit die Disharmonie in der Außenpolitik zwischen ihm und der Regierung bemerkbar. Am 14. Oktober 2008 eskalierte der Konflikt zwischen Tusk und Kaczyński medienwirksam. Um die Anwesenheit des Präsidenten beim EUGipfel in Brüssel zu verhindern, wurde dem Präsidenten von der Regierungskanzlei die Benutzung des Regierungsflugzeugs untersagt. Tusk hatte vor der Entscheidung gestanden, sich entweder auf Zugeständnisse einzulassen, um eventuell eine friedliche Koexistenz erreichen zu können, oder heftigen Streit und politische Konflikte in Kauf zu nehmen, um seine Regierungsprärogativen zu verteidigen. Lech Kaczyński erschien jedoch trotz gegenteiliger Bemühungen der Regierungsmitarbeiter auf dem EU-Gipfel, denn seine Kanzlei hatte für ihn ein anderes Flugzeug gemietet, und nutzte darüber hinaus die misslungene Regierungsaktion, um einen gewaltigen Medienskandal zu inszenieren.

Nach einem nur scheinbaren und kurzfristigen Tauwetter im Zusammenspiel zwischen den beiden Politikern, das auf die Notwendigkeit der Zusammenarbeit gegen die Weltwirtschaftskrise zurückzuführen war, folgte von Seiten des Präsidenten erneut starke Kritik an der Regierungspolitik, die er, ähnlich wie die PiS, als viel zu passiv bewertete, um den Folgen der Krise effektiv entgegenwirken zu können.

Im März 2009 versuchten beide Politiker nach einem gemeinsamen »Ausflug nach Brüssel« die dort beim EUGipfel erzielten Erfolge der eigenen Person zuzuschreiben. Jedoch schon zwei Wochen später sollte sich erneut bemerkbar machen, wie schädlich der Konkurrenzkampf zwischen den beiden Machtorganen sein und welche negativen Folgen die mangelnde Zusammenarbeit zwischen ihnen für die polnische Außenpolitik haben kann. Durch den unzureichenden Informationsaustausch zwischen den beiden Kanzleien vor dem NATO-Gipfel im April 2009 verlor Außenminister Radosław Sikorski nicht nur jede Möglichkeit, zum Generalsekretär ernannt zu werden. Seine Chancen waren ohnehin eher gering, jedoch hätte durch strategische Koordination zwischen der Regierung und dem Präsidenten mehr aus dieser Situation gemacht werden können. Die Regierung erwartete für die Zustimmung der Kandidatur des dänischen Ministerpräsidenten Rasmussen ein NATO-Fernmeldebataillon und zumindest einige weniger wichtige Posten im NATO-Apparat. Im Nachhinein warfen sich beide Seiten gegenseitig die Schuld an der Situation vor. 


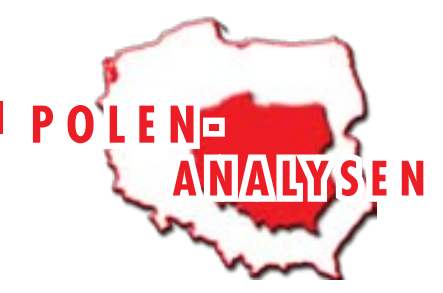

Auch einige Tage später stritten beide, Donald Tusk und Lech Kaczyński, beim EU-NATO-Gipfel nicht nur um den Anspruch auf die Formulierung der Vorgaben für die politische Stellungnahme, sondern auch darum, wer sich mit US-Präsident Barack Obama treffen dürfe. Da keiner von ihnen nachgeben wollte, musste ein gemeinsames Treffen aller drei Politiker organisiert werden.

\section{Urteil des Verfassungsgerichts vom 20. Mai 2009}

Infolge des zuvor beschriebenen Konflikts um das Recht, Polen bei wichtigen EU-Gipfeln in Brüssel zu vertreten, stellte Donald Tusk bereits im Oktober 2008 beim Verfassungstribunal den Antrag, das zentrale Staatsorgan zu bestimmen, das dazu berechtigt sei, die Republik Polen während der Sitzungen des Europäischen Rates zu vertreten und damit das Land zu repräsentieren. Um den Kompetenzstreitigkeiten zwischen den beiden Machtzentren ein Ende zu setzen, sprach das Verfassungsgericht am 20. Mai 2009 folgendes Urteil:

1. Laut Präambel und Art. 133 Abs. 3 sollen der Präsident, der Ministerrat und der Ministerpräsident bei der Ausführung ihrer Verfassungskompetenzen zusammenwirken.

2. Der Präsident als oberster Vertreter der Republik Polen kann laut Art. 126 Abs. 1 die Entscheidung treffen, an der konkreten Sitzung des Europäischen Rates teilzunehmen, wenn er dies als zweckmäßig für die Ausführung seiner im Art. 126 Abs. 2 bestimmten Aufgaben erkennt.

3. Laut Art. 146 Abs. 1,2 und 4 wird die Stellungnahme der Republik Polen vom Ministerrat festgelegt. Der Ministerpräsident vertritt die Republik Polen und präsentiert den festgelegten Standpunkt während der Sitzung des Europäischen Rates.

4. Für die Teilnahme des Präsidenten an der Sitzung des Europäischen Rates ist sein Zusammenwirken mit dem Ministerpräsidenten und dem entsprechenden Minister erforderlich, so wie es im Art. 133 Abs. 3 festgelegt ist, um die Einheitlichkeit der Handlungen gegenüber der EU und ihren Institutionen zu gewährleisten.

5. Das Zusammenwirken des Präsidenten mit dem Ministerpräsidenten und dem entsprechenden Minister gibt ihm die Möglichkeit, sich in Angelegenheiten, die mit der Ausführung seiner Aufgaben laut Art.126 Abs. 2 verbunden sind, zu der vom Ministerrat festgelegten Stellungnahme zu äußern. Er hat auch die Möglichkeit, den Umfang und die Form der Teilnahme des Präsidenten an der konkreten Sitzung des Europäischen Rates zu präzisieren.
Der Beschluss des Verfassungstribunals bedeutet also, dass Lech Kaczyński in Zukunft immer dann an den Sitzungen des Europäischen Rates teilnehmen kann, wenn er dies für richtig hält, die Richtung der Europapolitik jedoch eindeutig von der Regierung bestimmt wird und der Präsident dann auch die Vorgaben des Ministerrates zu befolgen hat.

\section{Die Vetostrategie des Präsidenten}

Aber nicht nur in der Außenpolitik gestaltet sich das Verhältnis zwischen Lech Kaczyński und Donald Tusk mehr als problematisch. Mit seinem Vetorecht verfügt der Präsident über ein sehr wirksames Instrument, um der Regierung "Sand ins Getriebe zu streuen«.

Die wichtigsten innenpolitischen Bereiche, in denen die Reformpolitik der Regierung durch den Präsidenten schmerzhaft gehemmt werden kann, sind Fragen der Privatisierung, des Rentensystems sowie des Gesundheitsund Bildungswesens. Selbstverständlich kann dabei nicht davon ausgegangen werden, dass der Präsident den Regierungsvorschlägen stets enthusiastisch gegenüber steht. Dennoch ist es für den politischen Prozess unabdingbar, dass Debatten sachlich und zielgerichtet geführt werden und nicht an Emotionen, persönlichen Animositäten oder politischen Strategien scheitern.

Entgegen den anfänglichen Deklarationen bestand die Taktik von Lech Kaczyński vor dem Hintergrund der 2010 bevorstehenden Präsidentschaftswahlen darin, kleinere Gesetzesvorhaben zu unterschreiben und bei den für die Effektivität der Regierungspolitik relevanten Reformvorschlägen von seinem Vetorecht Gebrauch zu machen. Um das präsidenzielle Veto zurückzuweisen, sind 3/5 der Stimmen im Sejm nötig, deshalb brauchte die Koalition von $P O$ und $P S L$ immer entweder Stimmen der PiS, auf die sie aber natürlich nicht zählen konnte, oder sie war auf die oppositionelle Demokratische Linksallianz (Sojusz Lewicy Demokratycznej-SLD) angewiesen, die aber um den Aufbau ihres eigenen politischen Images einer sozialdemokratischen Partei bemüht war und deshalb meist kein ausgeprägtes Interesse daran hatte, die Politik der Regierung zu unterstützen.

Bereits beim zweiten Veto des Präsidenten in der laufenden Legislaturperiode wurde aufgrund der fehlenden qualifizierten Mehrheit zu seiner Zurückweisung das Änderungsgesetz über den Rundfunk und das Fernsehen blockiert, was die Absetzung des durch die ehemalige Regierung politisierten Vorstandes in den öffentlichen Medien unmöglich machte. Durch ein weiteres Veto wurde die Durchführung von Privatisierungsvorhaben in Polen gestoppt. Bisher wurden mit den 18 eingelegten Vetos außerdem u. a. Reformen im 


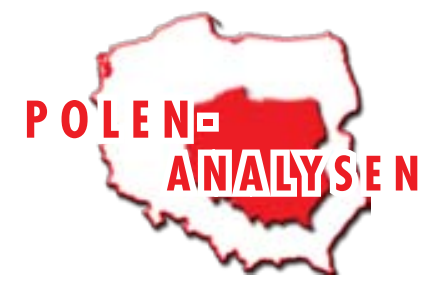

Gerichtswesen und Rentensystem, aber vor allem drei Gesetze zur Reform des Gesundheitswesens blockiert, welche die Rettung vieler hoch verschuldeter Krankenhäuser ermöglichen sollten. In seiner Begründung bediente sich der Präsident des populistischen Arguments, wonach in "Polen als dem Land mit gesellschaftlicher Solidarität« die Privatisierung von Krankenhäusern verhindert werden müsse, um der kapitalistischen Kommerzialisierung des Gesundheitswesens im Allgemeinen vorzubeugen.

Zu einem der jüngsten Erfolge Lech Kaczyńskis muss sein Einspruch gegen das von der Regierungskoalition mit anfänglicher Unterstützung durch die $S L D$ vorgelegte Mediengesetz gezählt werden. In dem Gesetz sollte eine ganz neue Finanzierungspraxis der öffentlichen Medien verankert werden, die u. a. die Abschaffung der Rundfunkgebühren und die Finanzierung der öffentlichen Medien aus dem Staatshaushalt vorsah. Außerdem sollte dabei auf die direkte Finanzierung der Medieninstitutionen verzichtet und diese durch die Finanzierung von bestimmten Aufgaben ersetzt werden. Darüber hinaus sollte eine stärkere Regionalisierung durchgesetzt werden, indem die einzelnen Regionalanstalten zu unabhängigen Gesellschaften umgestaltet werden sollten. Nachdem jedoch vom Senat Änderungsvorschläge eingebracht wurden, durch die u. a. die Bestimmung eines Minimalbetrages für die Finanzierung der öffentlichen Medien aus dem Staatshaushalt entfiel und ihr Aufgabenkatalog um die Förderung von christlichen Werten erweitert wurde, verlor das Projekt die Unterstützung der SLD. Nachdem sich die PiS, die Linke (Lewica) sowie die parlamentarischen Gruppen Polen XXI (Polska $X X I$ ), Sozialdemokratie Polens (Socjaldemokracja Polski$S d P l$ ) und Demokratische Partei (Stronnictwo Demokratyczne - SD) für die Aufrechterhaltung des Präsidentenvetos ausgesprochen hatten, wurde dieser Gesetzesentwurf abgelehnt.

Erfolglos war im Gegensatz dazu das Veto des Präsidenten zur Verhinderung des Gesetzes gegen die von der PO bereits im Wahlkampf 2007 anvisierte Trennung der Funktionen des Justizministers und des Generalstaatsanwalts. Die Notwendigkeit einer Trennung beider Funktionen wurde u. a. bei der kontroversen Amtsführung von Zbigniew Ziobro, Justizminister in der Regierung von Jaroslaw Kaczyński, sichtbar, dem eine Politisierung der Staatsanwaltschaft vorgeworfen worden war. Aus diesem Grund unterstützte auch die oppositionelle $S L D$ diesen Gesetzesentwurf, so dass das Präsidentenveto im Oktober dieses Jahres abgewiesen wurde und das Gesetz am 31. März 2010 in Kraft treten wird.

\section{Die gesellschaftliche Wahrnehmung des Konflikts}

Als einer der wichtigen Indikatoren zur Analyse des Konflikts zwischen Donald Tusk und Lech Kaczyński kann die gesellschaftliche Unterstützung der beiden Politiker dienen. Bei den Umfragen von CBOS (Zentrum für Erforschung der gesellschaftlichen Meinung) fällt auf, dass die Amtsführung von Lech Kaczyński von der Bevölkerung stets sehr kritisch beurteilt wird. Im Juni 2009 bewerteten nur 22\% der Befragten die politischen Aktivitäten des Präsidenten als "gut", dabei wurde seine Politikführung von $68 \%$ der Respondenten als "eher schlecht« oder "sehr schlecht» angesehen. Selbst $45 \%$ der Wähler, die Lech Kaczyński bei der Präsidentschaftswahl 2005 ihre Stimme gegeben hatten, stehen seiner Art der Amtsausübung mittlerweile kritisch gegenüber. Dies stellt sich bei Donald Tusk ganz anders dar. Obwohl auch seine Werte kontinuierlich sinken, ist die Zahl der mit ihm als Ministerpräsident zufriedenen Bürger (46\%) immer noch entschieden größer als die derjenigen, die unzufrieden sind (38\%).

Auch die Konflikte zwischen den beiden Machtzentren werden von der Gesellschaft sehr kritisch aufgenommen. Bereits seit Anfang 2008 ist eine große Mehrheit der polnischen Bevölkerung der Meinung, dass die Zusammenarbeit zwischen dem Präsidenten und dem Ministerpräsidenten schlecht funktioniert. Im Januar 2009 waren es $90 \%$ der Befragten. Dabei vertraten $60 \%$ der Bürger die Meinung, dass Lech Kaczyński zu oft von seinem Vetorecht Gebrauch mache; nur 25\% hielten dies für angemessen.

Aufgrund dieser kritischen Wahrnehmung des Kohabitationskonflikts zwischen den beiden Exekutivorganen sehen $68 \%$ der Bevölkerung die Notwendigkeit der Verfassungsänderung im Bereich der Kompetenzverteilung zwischen dem Präsidenten und der Regierung. Dabei haben sich $65 \%$ der Befürworter der Verfassungsänderung für mehr Prärogativen zugunsten der Regierung ausgesprochen und nur $30 \%$ wollten die Position des Präsidenten stärken. Bei der Frage nach der besten bzw. erstrebenswerten demokratischen Regierungsform sprachen sich die meisten Befragten jedoch weiterhin für das bestehende Regierungssystem mit einer doppelköpfigen Exekutive aus. Ein parlamentarisches Kabinettssystem würde bei $32 \%$ der Bürger Unterstützung finden; ein politisches System, in dem der Präsident die meisten Machtbefugnisse hätte, befürworteten nur $13 \%$ der Befragten.

\section{Fazit}

Auch im Ausland wird der Konflikt zwischen Präsident und Regierung zunehmend negativ wahrgenommen, 


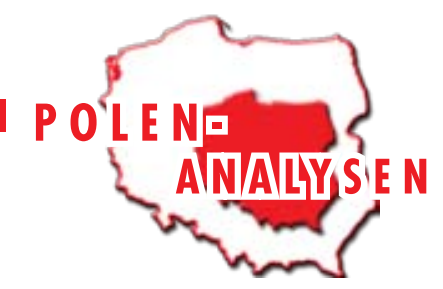

wodurch die außenpolitische Position Polens eindeutig geschwächt wird. Wie die bisherige Praxis zeigt, können diese Konflikte, Missverständnisse und falsch verstandenen Ambitionen von außenpolitischen Partnern wie den USA und den EU-Partnern oder auch von außenpolitischen Gegnern sehr gut ausgespielt werden. Auch die innenpolitische Effektivität leidet darunter, dass an die Stelle einer Zusammenarbeit zwischen den beiden Organen der polnischen Exekutive eine ständige, zermürbende Konfrontation tritt, wodurch der Gesetzgebungsprozess insbesondere in Bezug auf wichtige Reformen erheblich gehemmt wird.

Zurzeit ist allerdings kein Ende der konfliktträchtigen Konstellation der Kohabitation abzusehen. Als aktuelles Beispiel lassen sich die erneuten Spannungen um die Nominierung der Botschafter nennen. Als besonders problematisch können sich in Zukunft Konfliktpotenziale erweisen, die die Prärogativen im Bereich der Streitkräfte betreffen. Ein Beweis für die politische Instrumentalisierung der wichtigsten staatlichen Angelegenheiten ist auch die Zusammensetzung des Rates für die Nationale Sicherheit beim Präsidenten, der alles andere als unparteiisch ist und aus dem PiS-Vorsitzenden Jarosław Kaczyński sowie dessen ehemaligen Ministern Anna Fotyga (Außenministerin) und Aleksander Szczygło (Verteidigungsminister) besteht. Auch wenn der Rat nicht sehr oft tagt, hat Jarosław Kaczyński dadurch Zugang zu allen Staatsgeheimnissen, wodurch die Zusammensetzung des Rates auf die Existenz eines "Schattenkabinetts" hindeuten könnte. Die Position von Lech Kaczyński scheint in den letzten Wochen jedoch nicht nur in der gesellschaftlichen Wahrnehmung, sondern auch wegen der mangelnden Loyalität seiner Mitarbeiter geschwächt. Aufgrund der in einem Interview offen geäußerten Kritik an seinem Arbeitgeber musste Piotr Kownacki von seinem Amt als Leiter der Präsidialkanzlei zurücktreten.

Vor diesem Hintergrund werden von verschiedenen Seiten (u. a. Verfassungsrechtler und Politiker) bestimmte Verfassungsänderungen für sinnvoll erachtet, durch die in der Zukunft die Turbulenzen zwischen den exekutiven Machtzentren verhindert werden könnten. Im Moment ist jedoch keiner der beiden Hauptakteure daran interessiert, die Verfassungsbausteine neu zusammenzulegen. Lech Kaczyński wird keine Kompetenzen abgeben wollen und Donald Tusk, der trotz der jüngsten »Glücksspielaffäre« im Regierungslager darauf hofft, aus den nächsten Präsidentschaftswahlen als Sieger hervorzugehen, hat auch kein Interesse daran, zum jetzigen Zeitpunkt an der Verfassung zu werkeln. In der aktuellen politischen Konstellation wird also eine Verfassungsänderung nicht möglich sein, und es bleibt nur, sich auf weitere Konflikte einzustellen und den Ausgang der kommenden Präsidentschaftswahlen abzuwarten.

\section{Über die Autoren}

Artur Kopka ist Wissenschaftlicher Mitarbeiter und Dozent an der Europa-Universität Viadrina Frankfurt (Oder) am Lehrstuhl Politikwissenschaft: Vergleichende Analyse politischer Systeme, Bewegungen und Kulturen. Als Stipendiat des DAAD promoviert er zum Thema: »Polen 2005-2007: rechtspopulistischer Rückschlag oder weitere demokratische Konsolidierung?«. Seine Forschungsinteressen liegen in den Bereichen Zeitgeschichte und Demokratieentwicklung in Polen, politische Kultur in den Posttransformationsgesellschaften, gesellschaftliche Integrationsprozesse in der deutsch-polnischen Grenzregion.

Claudia Schäfer ist Wissenschaftliche Angestellte und Dozentin am Seminar für Wissenschaftliche Politik der Universität Freiburg. 2008 erschien ihre Magisterarbeit unter dem Titel »Semi-Präsidentialismus à la polonaise - Zum Einfluss des Regimetyps auf die demokratische Konsolidierung in der Republik Polen« im Ergon-Verlag Würzburg. Als Stipendiatin des Evangelischen Studienwerkes Villigst promoviert sie zum Thema "Europäisierung in Ostmitteleuropa: Subnationale Effekte von Konditionalität und Mitgliedschaft«. 


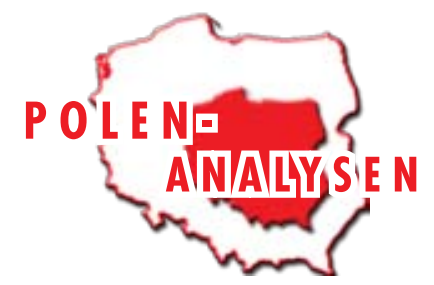

\section{Tabellen und Grafiken}

\section{Umfragen zur Kohabitation in Polen nach 2007}

Grafik 1: Wie bewerten Sie die Tätigkeit von Staatspräsident Lech Kaczyński?

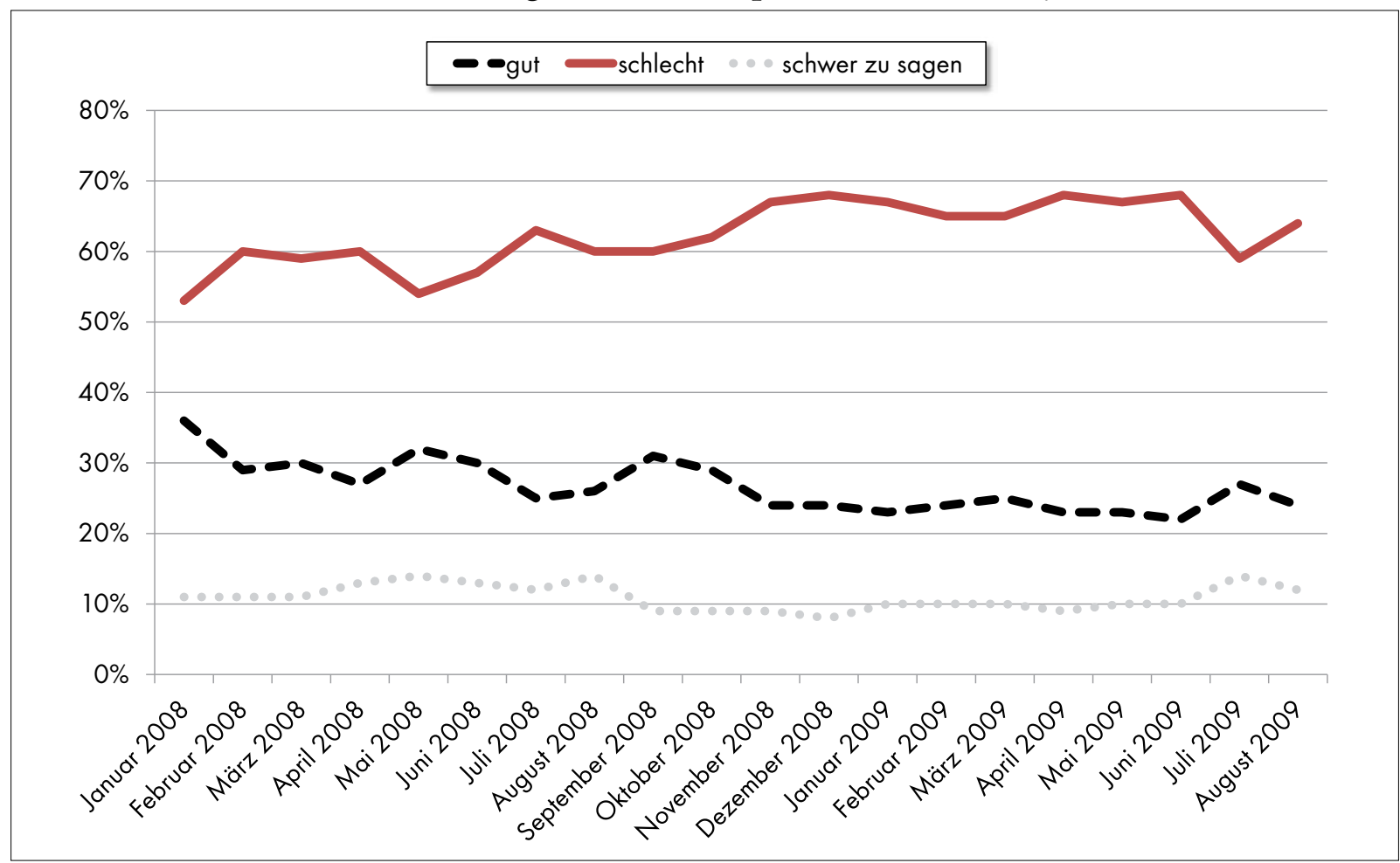

\begin{tabular}{|l|c|c|c|c|c|c|c|c|}
\hline & Jan. 2008 & Feb. 2008 & März 2008 & April 2008 & Mai 2008 & Juni 2008 & Juli 2008 & Aug. 2008 \\
\hline gut & $36 \%$ & $29 \%$ & $30 \%$ & $27 \%$ & $32 \%$ & $30 \%$ & $25 \%$ & $26 \%$ \\
schlecht & $53 \%$ & $60 \%$ & $59 \%$ & $60 \%$ & $54 \%$ & $57 \%$ & $63 \%$ & $60 \%$ \\
\hline schwer zu sagen & $11 \%$ & $11 \%$ & $11 \%$ & $13 \%$ & $14 \%$ & $13 \%$ & $12 \%$ & $14 \%$ \\
\hline
\end{tabular}

\begin{tabular}{|l|c|c|c|c|c|c|c|c|}
\hline & Sept. 2008 & Okt. 2008 & Nov. 2008 & Dez. 2008 & Jan. 2009 & Feb. 2009 & März 2009 & April 2009 \\
gut & $31 \%$ & $29 \%$ & $24 \%$ & $24 \%$ & $23 \%$ & $24 \%$ & $25 \%$ & $23 \%$ \\
schlecht & $60 \%$ & $62 \%$ & $67 \%$ & $68 \%$ & $67 \%$ & $65 \%$ & $65 \%$ & $68 \%$ \\
schwer zu sagen & $9 \%$ & $9 \%$ & $9 \%$ & $8 \%$ & $10 \%$ & $10 \%$ & $10 \%$ & $9 \%$ \\
\hline
\end{tabular}

\begin{tabular}{|l|c|c|c|c|}
\hline & Mai 2009 & Juni 2009 & Juli 2009 & Aug. 2009 \\
\hline gut & $23 \%$ & $22 \%$ & $27 \%$ & $24 \%$ \\
schlecht & $67 \%$ & $68 \%$ & $59 \%$ & $64 \%$ \\
schwer zu sagen & $10 \%$ & $10 \%$ & $14 \%$ & $12 \%$ \\
\hline
\end{tabular}

CBOS, BS/91/2009: Ocena działalności parlamentu i prezydenta [Bewertung der Tätigkeiten des Parlaments und des Präsidenten], Warszawa 06/2009 und CBOS, BS/114/2009: Ocena działalności prezydenta i parlamentu [Bewertung der Tätigkeiten des Staatspräsidenten und des Parlaments], Warszawa 08/2009, www.cbos.pl 


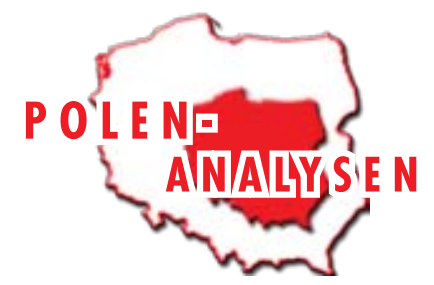

Grafik 2: Bewertung der Amtsführung von Staatspräsident Lech Kaczyński (spezifiziert nach Wählerschaft)

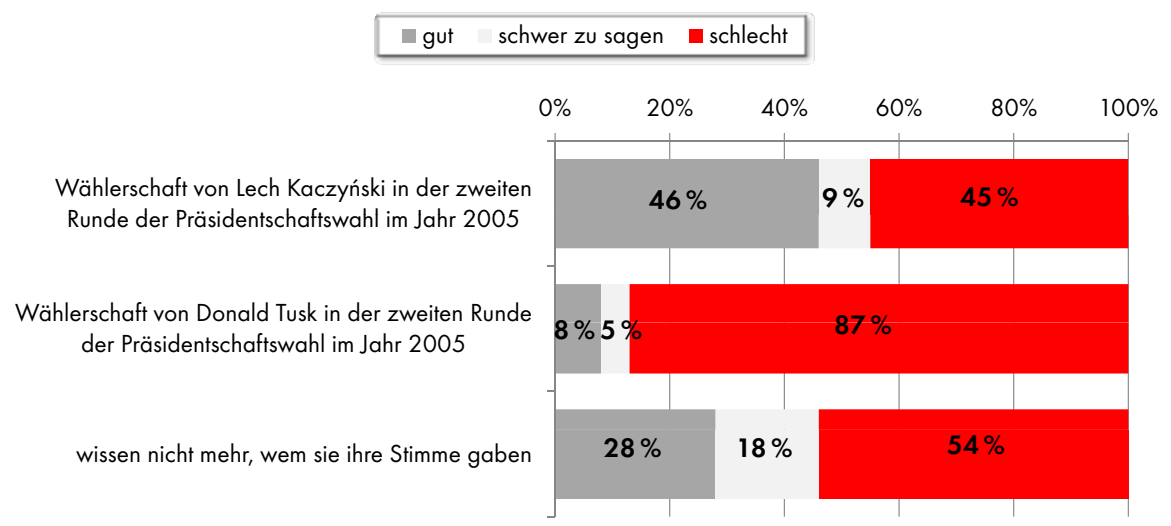

CBOS, BS/91/2009, Ocena dziatalności parlamentu i prezydenta [Bewertung der Tätigkeiten des Parlaments und des Staatspräsidenten], Warszawa 06/2009, www.cbos.pl

Grafik 3: Sind Sie zufrieden, dass Donald Tusk Regierungschef ist?

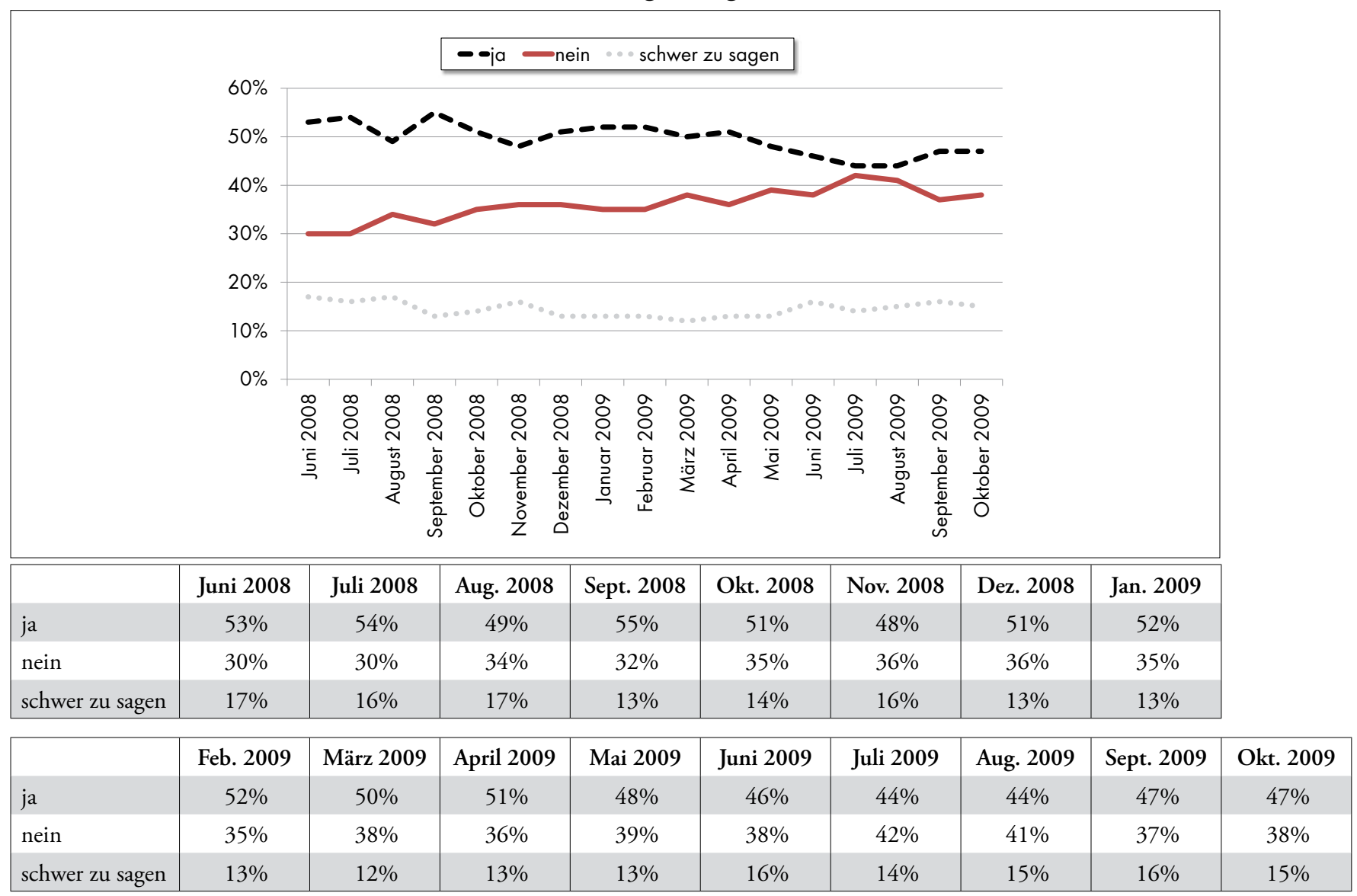

CBOS, BS/88/2009, Stosunek do rządu w czerwcu [Das Verhältnis zur Regierung im Juni], Warszawa 06/2009 und CBOS, BS/136/2009, Stosunek do rządu w październiku [Das Verhältnis zur Regierung im Oktober], Warszawa 10/2009, www.cbos.pl 


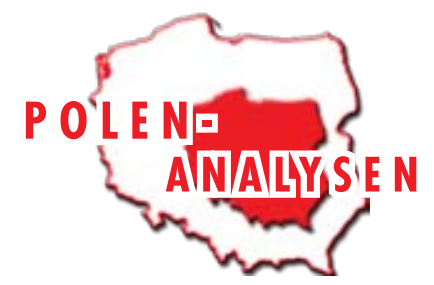

Grafik 4: Sind Sie zufrieden, dass Donald Tusk Regierungschef ist? (spezifiziert nach Wählerschaft)

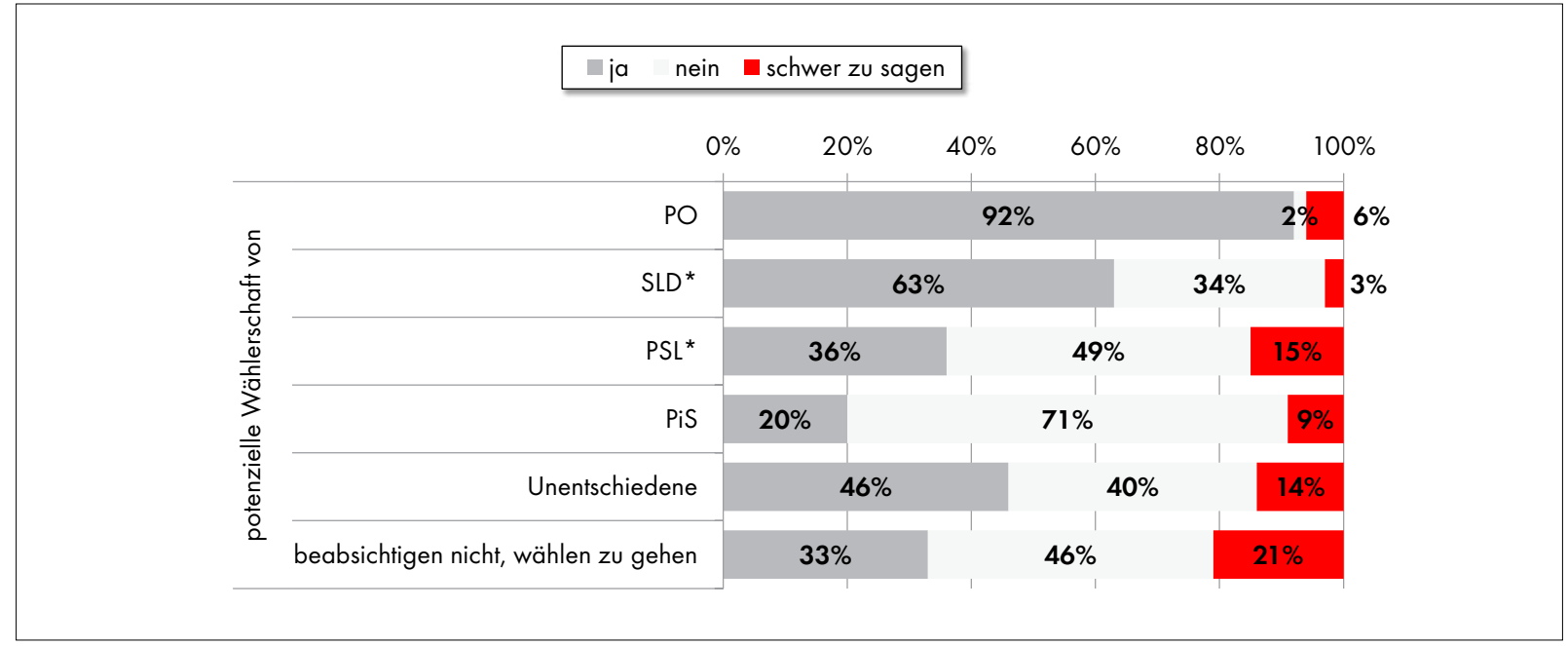

PO - Platforma Obywatelska - Bürgerplattform

SLD - Sojusz Lewicy Demokratycznej - Demokratische Linksallianz

PSL - Polskie Stronnictwo Ludowe - Polnische Bauernpartei

PiS - Prawo i Sprawiedliwość - Recht und Gerechtigkeit

* Daten zur Wählerschaft von SLD und PSL sollten mit Vorsicht interpretiert werden aufgrund der geringen Anzabl ihrer Anhänger in der Umfrage.

CBOS, BS/88/2009, Stosunek do rzadu w czerwcu [Das Verhältnis zur Regierung im Juni], Warszawa 06/2009, www.cbos.pl

Grafik 5: Wie funktioniert Ihrer Meinung nach die Zusammenarbeit zwischen der Regierung Tusk und Staatspräsident Lech Kaczyński?

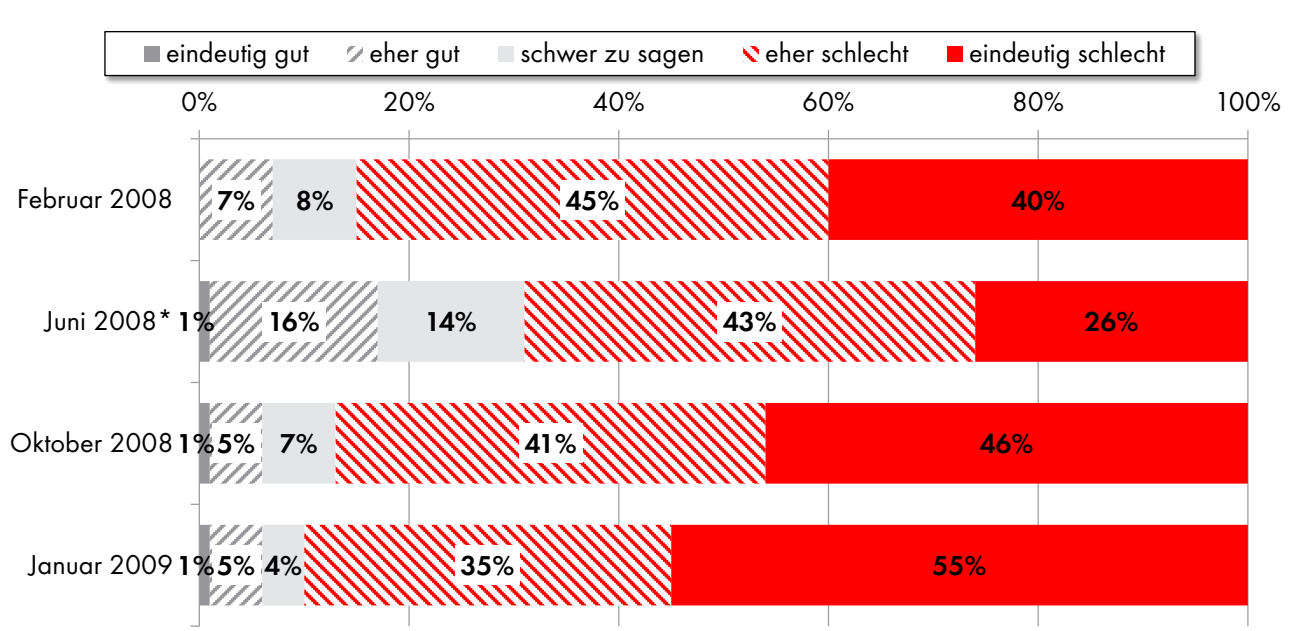

* Bei dieser Umfrage lautete die Frage: Wie beurteilen Sie die bisherige Zusammenarbeit von Präsident Lech Kaczyński mit der Regierung von Donald Tusk?

CBOS, BS/18/2009, Polacy o stosunkach między prezydentem a rządem [Die Polen über das Verhältnis zwischen dem Staatspräsidenten und der Regierung], Warszawa 02/2009, www.cbos.pl 


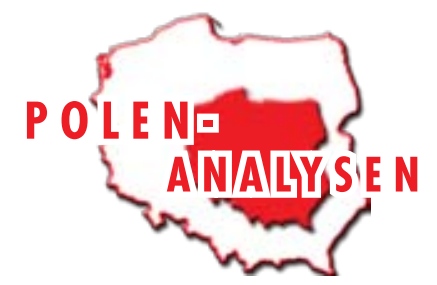

Grafik 6: Wie beurteilen Sie die Zusammenarbeit zwischen der Regierung Tusk und Staatspräsident Lech Kaczyński? (spezifiziert nach Wählerschaft)

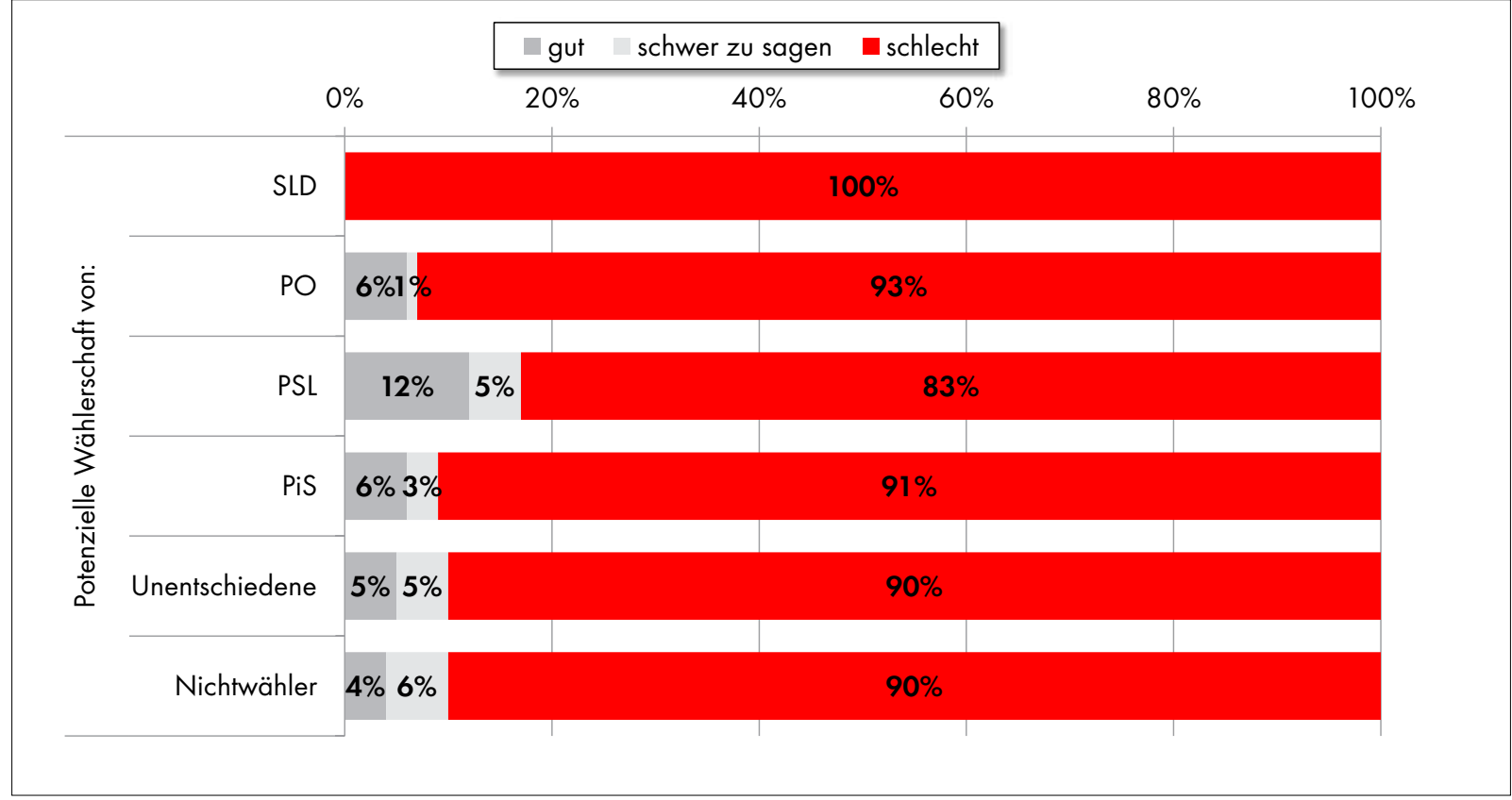

PO - Platforma Obywatelska - Bürgerplattform

SLD - Sojusz Lewicy Demokratycznej - Demokratische Linksallianz

PSL - Polskie Stronnictwo Ludowe - Polnische Bauernpartei

PiS - Prawo i Sprawiedliwość - Recht und Gerechtigkeit

CBOS, BS/18/2009: Polacy o stosunkach między prezydentem a rządem [Die Polen über das Verhältnis zwischen dem Staatspräsidenten und der Regierung], Warszawa 02/2009, www.cbos.pl

Grafik 7: Wenn Staatspräsident Lech Kaczyński Gesetze nicht unterschreibt, setzt er...

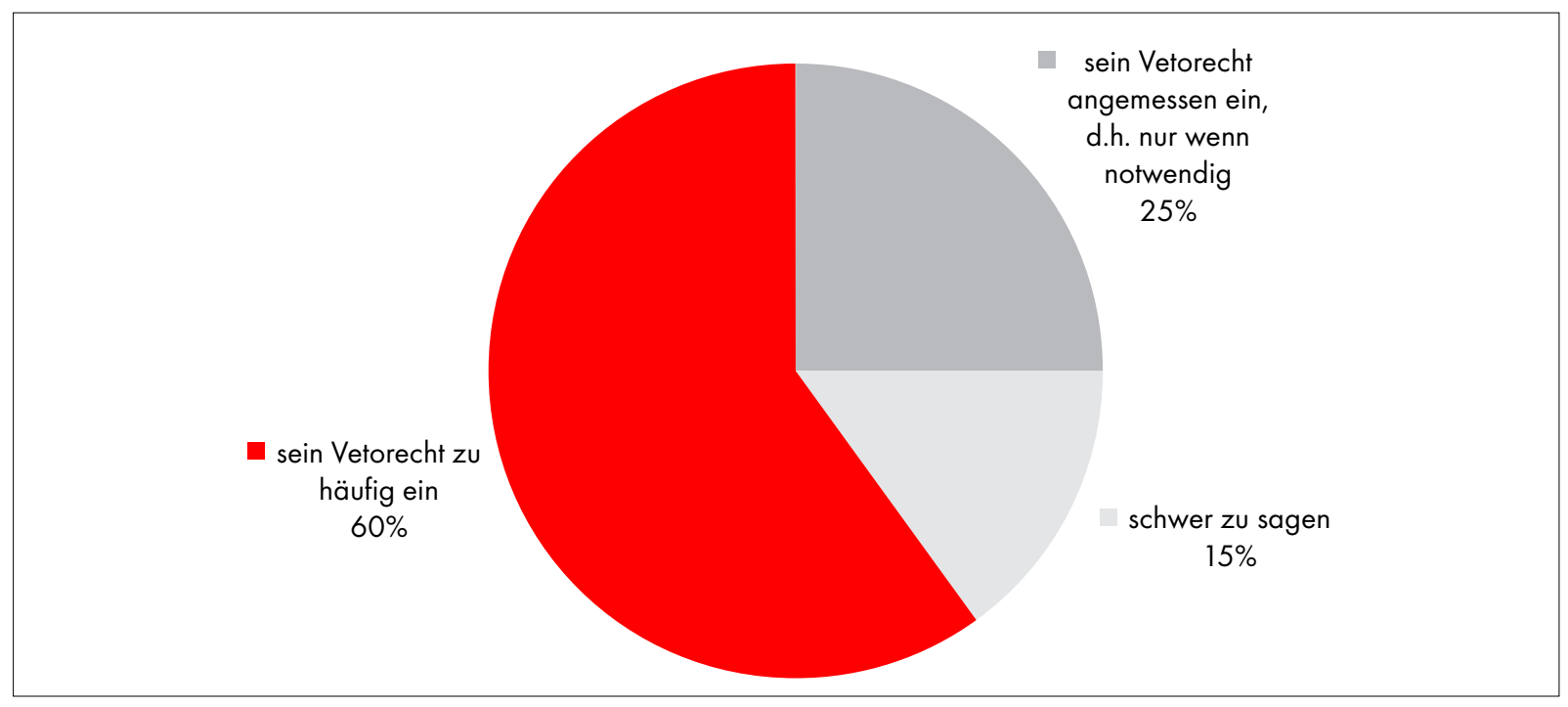

CBOS, BS/18/2009: Polacy o stosunkach między prezydentem a rządem [Die Polen über das Verhältnis zwischen dem Staatspräsidenten und der Regierung], Warszawa 02/2009, www.cbos.pl 


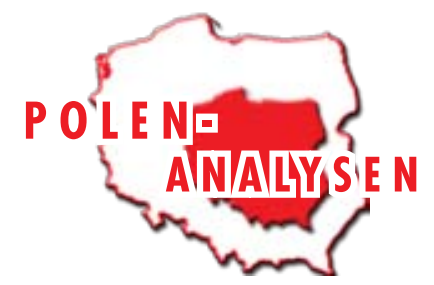

Grafik 8: Wenn Staatspräsident Lech Kaczyński Gesetze nicht unterschreibt, setzt er... (spezifiziert nach Wählerschaft*)

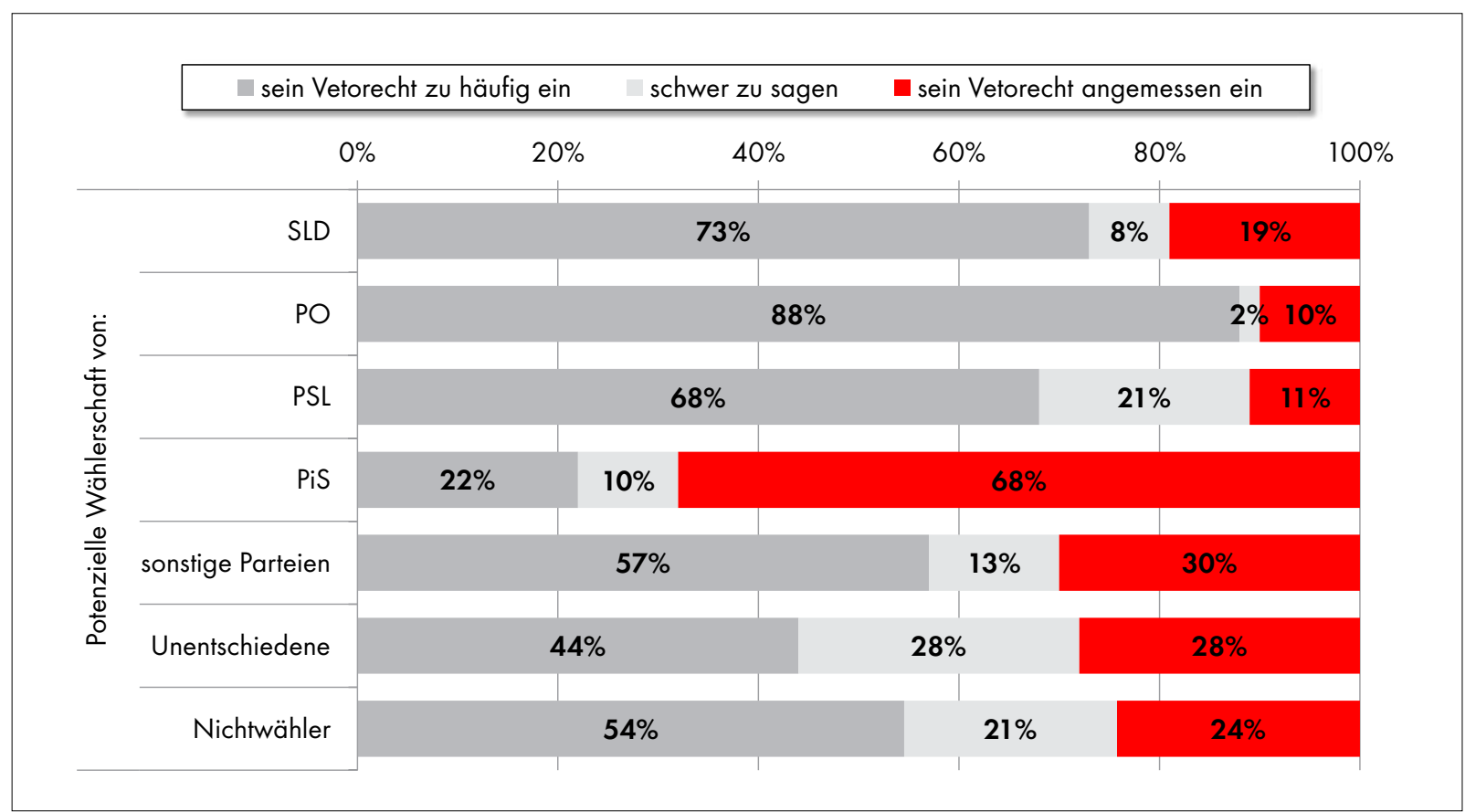

* Parteinamen siehe S. 11 .

CBOS, BS/18/2009: Polacy o stosunkach między prezydentem a rządem [Die Polen über das Verhältnis zwischen dem Staatspräsidenten und der Regierung], Warszawa 02/2009, www.cbos.pl

Grafik 9: Wenn Staatspräsident Lech Kaczyński Gesetze nicht unterschreibt, bemüht er sich vor allem, ...

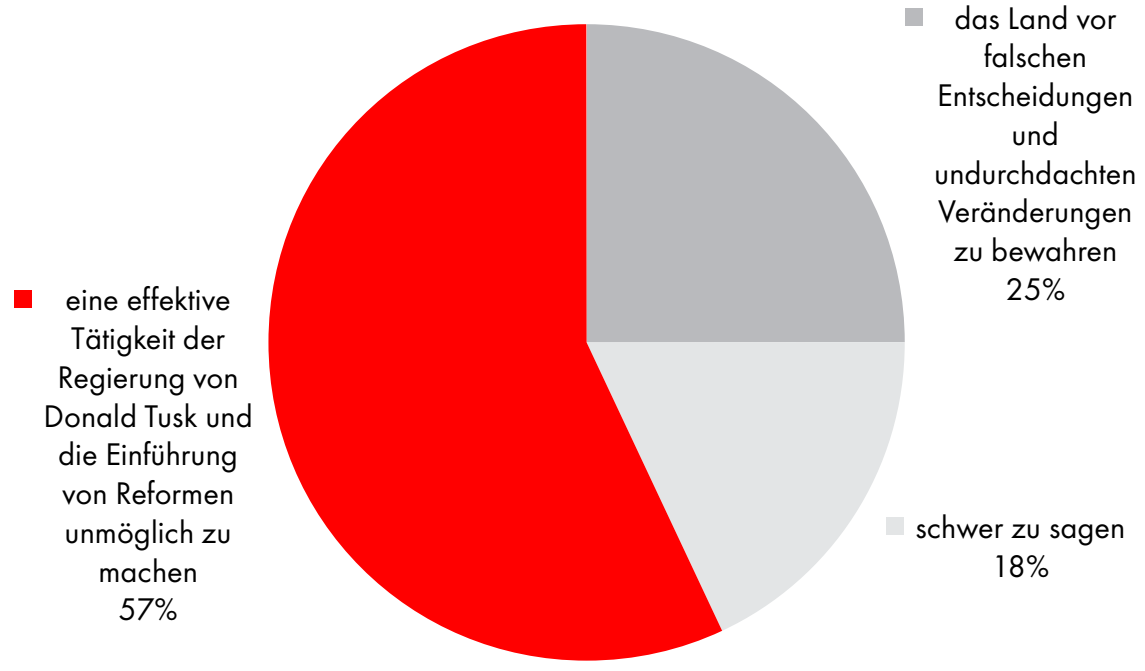

CBOS, BS/18/2009: Polacy o stosunkach między prezydentem a rządem [Die Polen über das Verhältnis zwischen dem Staatspräsidenten und der Regierung], Warszawa 02/2009, www.cbos.pl 


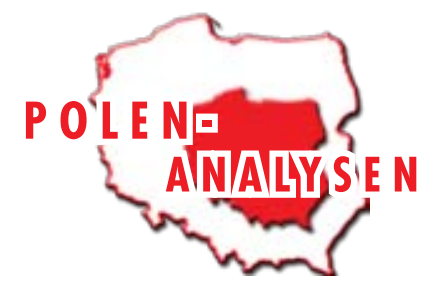

Grafik 10: Wenn Staatspräsident Lech Kaczyński Gesetze nicht unterschreibt, bemüht er sich vor allem, ... (spezifiziert nach Wählerschaft ${ }^{*}$ )

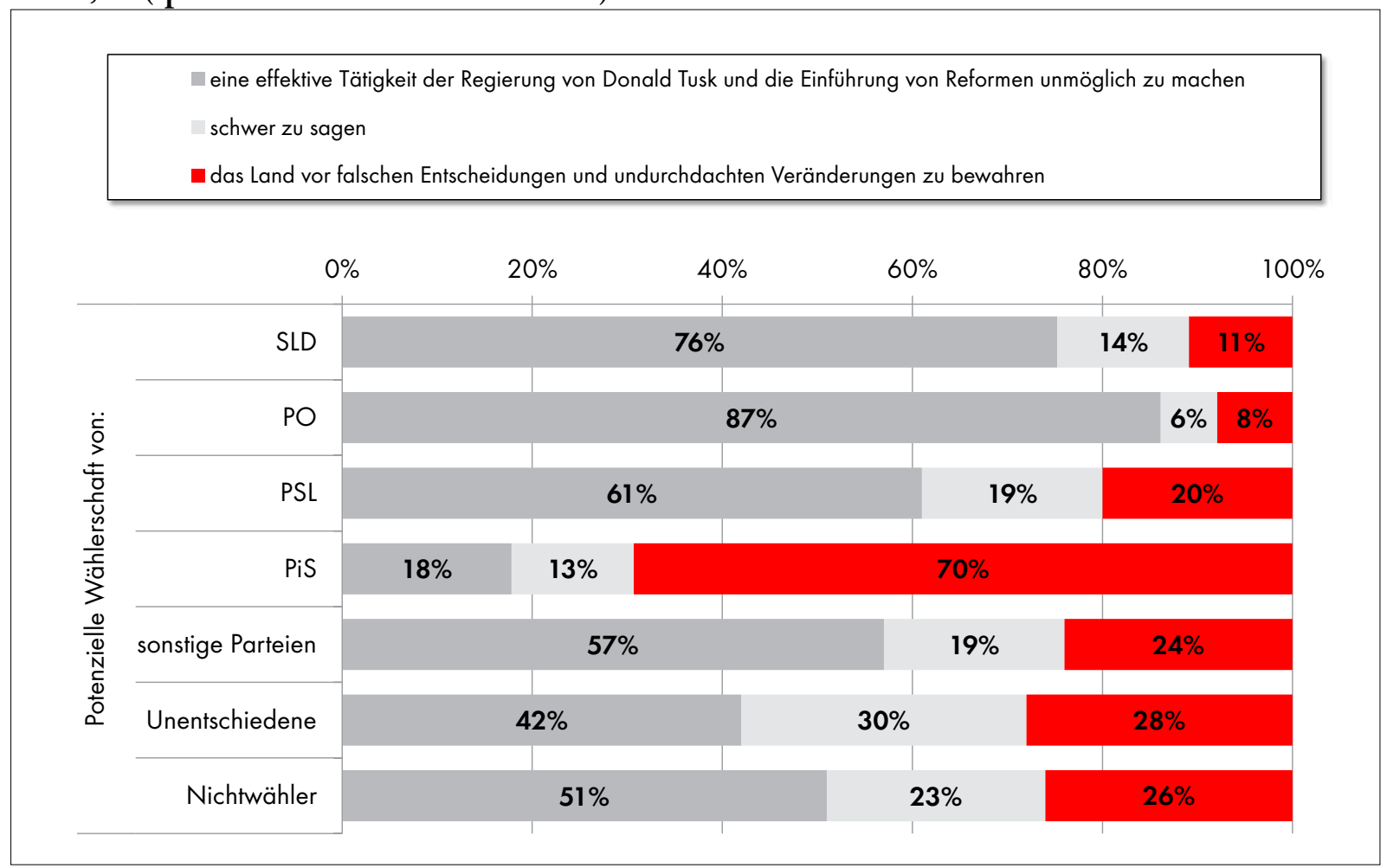

* Parteinamen siehe s. 11 .

CBOS, BS/18/2009: Polacy o stosunkach między prezydentem a rządem [Die Polen über das Verhältnis zwischen dem Staatspräsidenten und der Regierung], Warszawa 02/2009, www.cbos.pl

Grafik 11: Sollten in der Verfassung der Republik Polen Änderungen in der Kompetenzverteilung zwischen dem Staatspräsidenten und der Regierung vorgenommen werden?

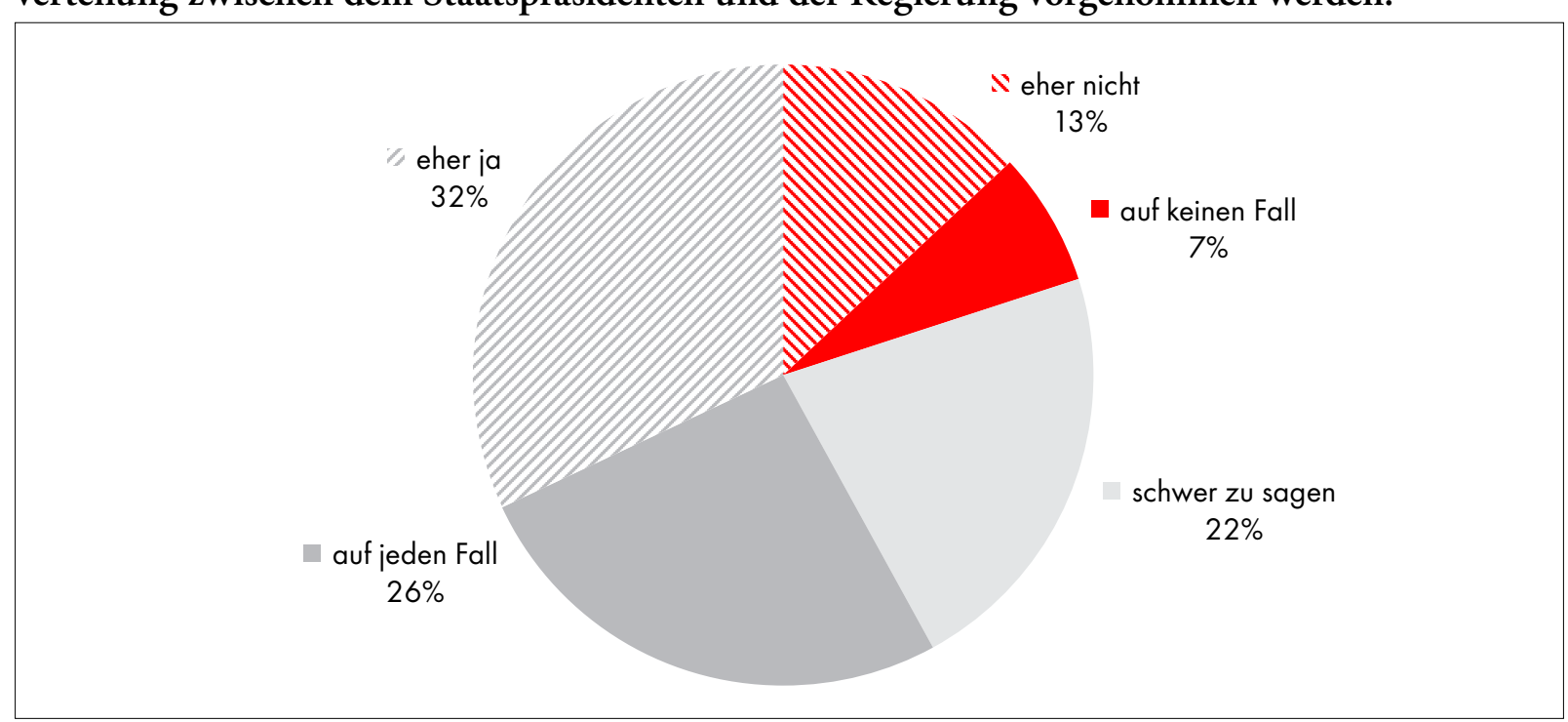

CBOS, BS/154/2008: Podziat kompetencji między prezydentem a premierem [Die Kompetenzverteilung zwischen dem Staatspräsidenten und dem Ministerpräsidenten], Warszawa 10/2008, www.cbos.pl 


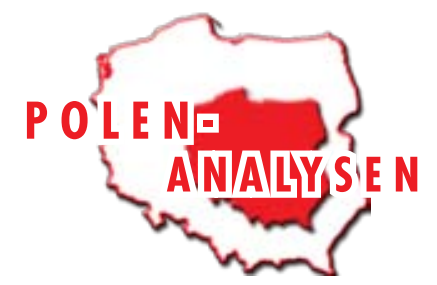

Grafik 12: Sollten in der Verfassung der Republik Polen Änderungen in der Kompetenzverteilung zwischen dem Staatspräsidenten und der Regierung vorgenommen werden? (\%) (spezifiert nach Wählerschaft)

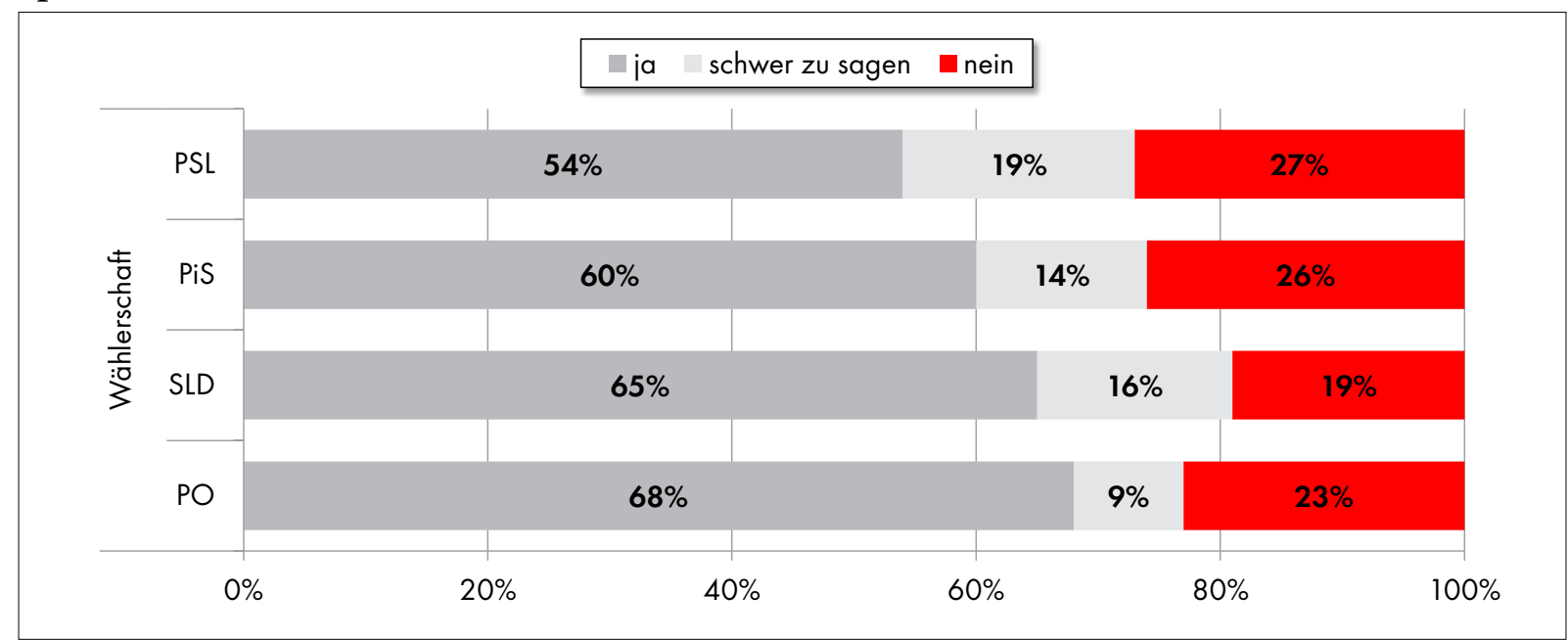

CBOS, BS/154/2008: Podziat kompetencji między prezydentem a premierem [Die Kompetenzverteilung zwischen dem Staatspräsidenten und dem Ministerpräsidenten], Warszawa 10/2008, www.cbos.pl

Grafik 13: Wer sollte mehr Kompetenzen als bisher haben? (Alle Befragten)

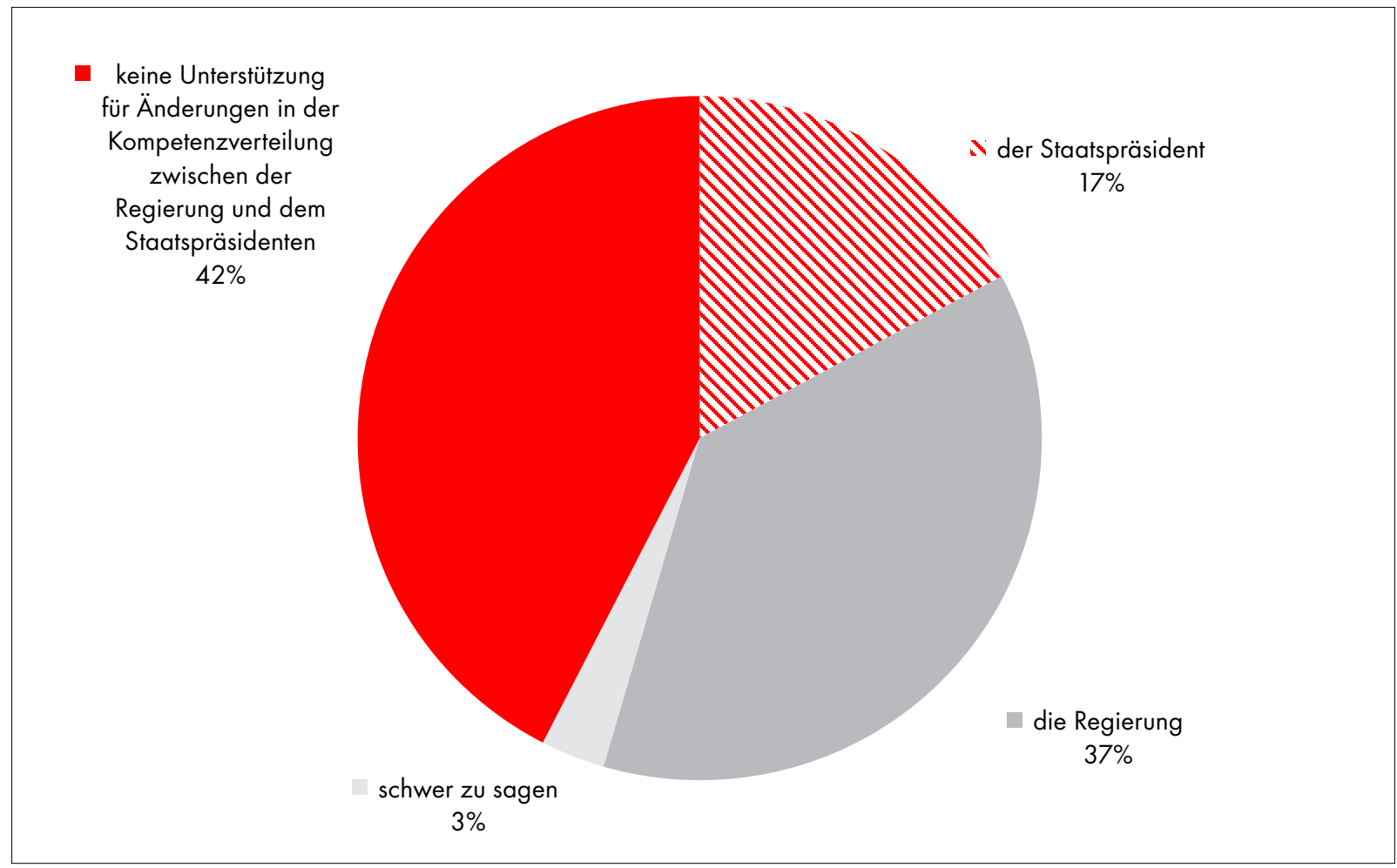

CBOS, BS/154/2008: Podziat kompetencji między prezydentem a premierem [Die Kompetenzverteilung zwischen dem Staatspräsidenten und dem Ministerpräsidenten], Warszawa 10/2008, www.cbos.pl 


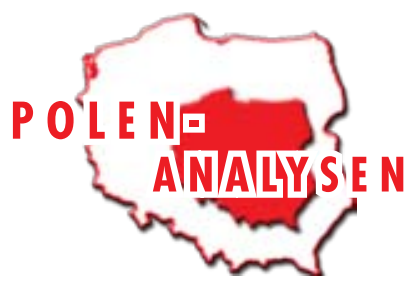

Grafik 14: Wer sollte mehr Kompetenzen als bisher haben? (Befürworter einer Verfassungsänderung)

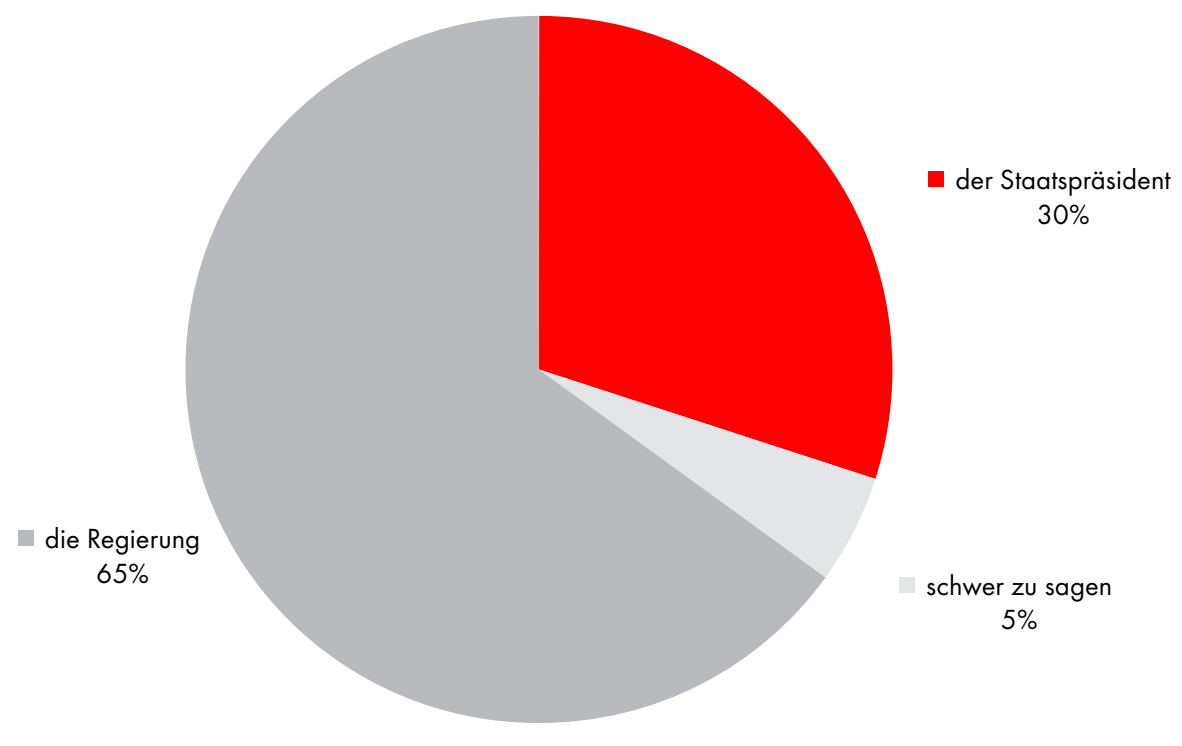

CBOS, BS/154/2008: Podziat kompetencji między prezydentem a premierem [Die Kompetenzverteilung zwischen dem Staatspräsidenten und dem Ministerpräsidenten], Warszawa 10/2008, www.cbos.pl

Grafik 15: Wer sollte mehr Kompetenzen als bisher haben? (spezifiert nach Wählerschaft)

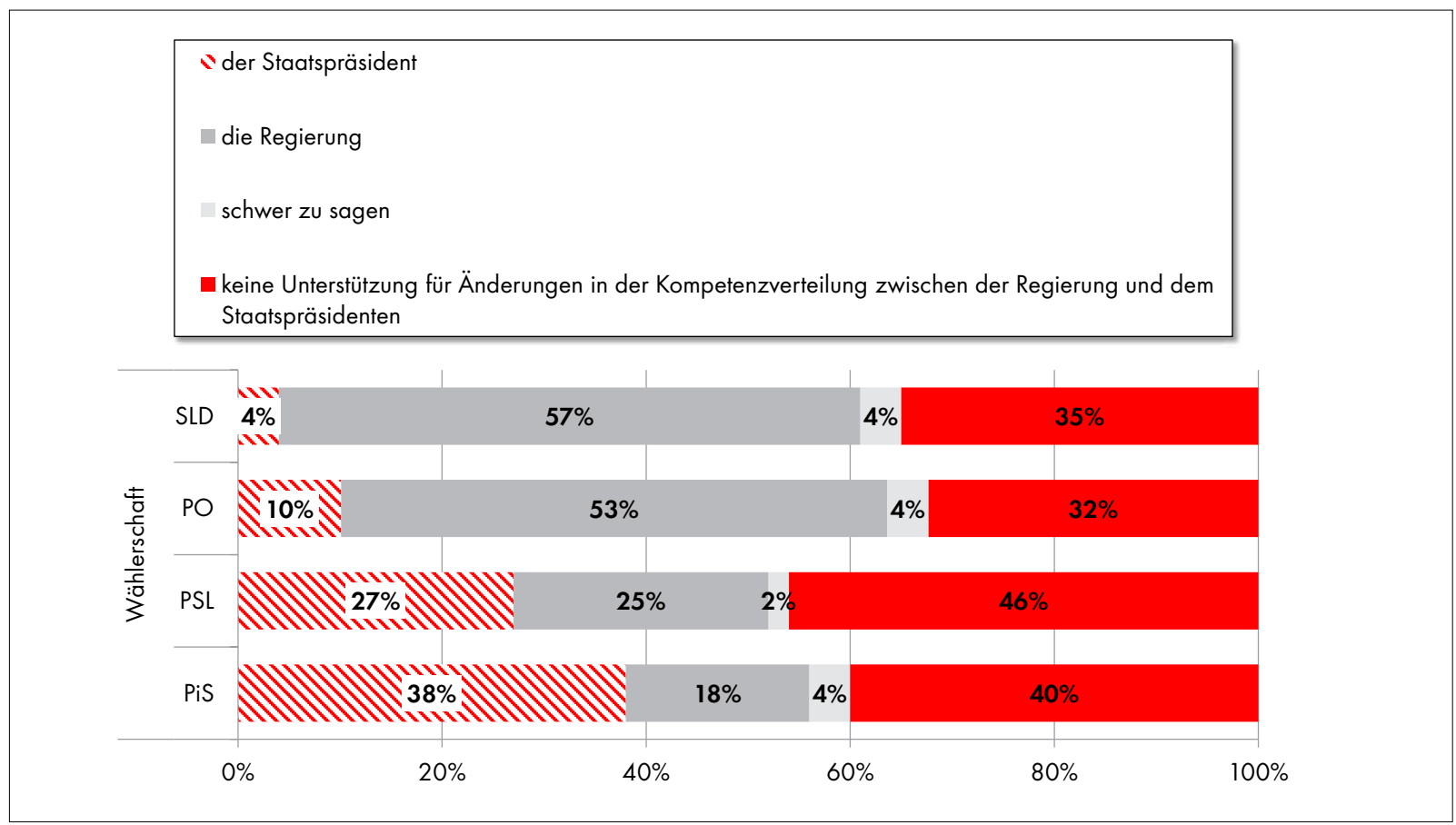

CBOS, BS/154/2008: Podział kompetencji między prezydentem a premierem [Die Kompetenzverteilung zwischen dem Staatspräsidenten und dem Ministerpräsidenten], Warszawa 10/2008, www.cbos.pl 


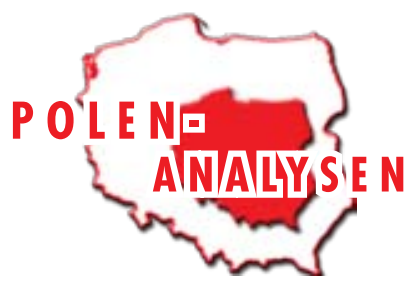

Grafik 16: Welches Regierungssystem sollte in Polen eingeführt werden?

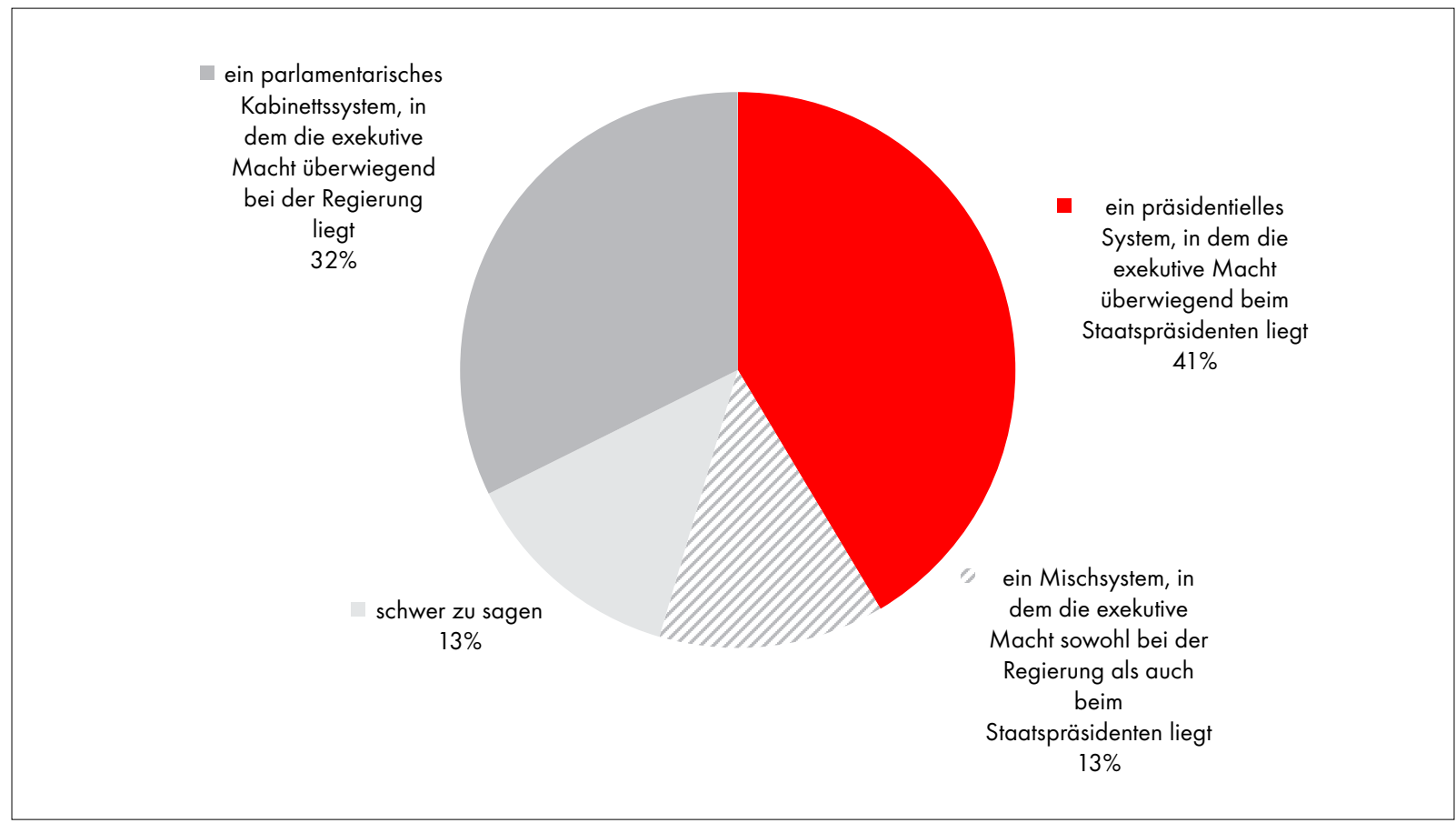

CBOS, BS/154/2008: Podziat kompetencji między prezydentem a premierem [Die Kompetenzverteilung zwischen dem Staatspräsidenten und dem Ministerpräsidenten], Warszawa 10/2008, www.cbos.pl

Grafik 17: Welches Regierungssystem sollte in Polen eingeführt werden? (spezifiziert nach Wählerschaft)

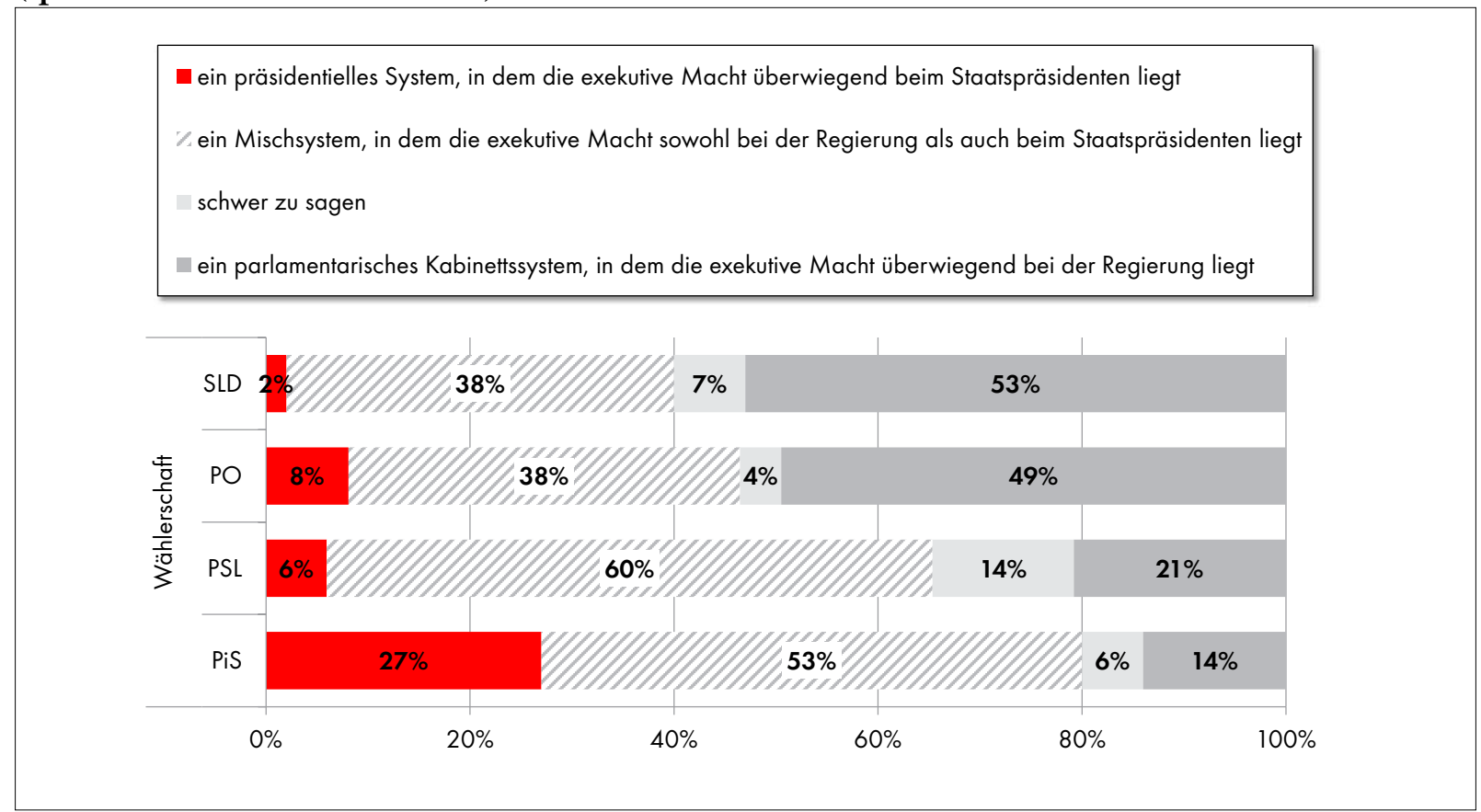

CBOS, BS/154/2008: Podziat kompetencji między prezydentem a premierem [Die Kompetenzverteilung zwischen dem Staatspräsidenten und dem Ministerpräsidenten], Warszawa 10/2008, www.cbos.pl 


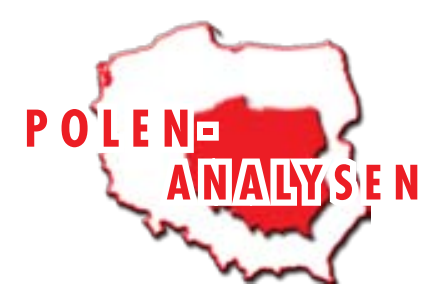

Chronik

\section{Vom 03. bis zum 16. November 2009}

\begin{tabular}{|c|c|}
\hline 03.11 .2009 & $\begin{array}{l}\text { Der Sejmausschuss für Gesetzgebung und öffentliche Finanzen beschließt, dem Antrag der Regierungspartei Bürger- } \\
\text { plattform (Platforma Obywatelska - PO) zur Einsetzung eines Untersuchungsausschusses zur sogenannten Glücks- } \\
\text { spielaffäre, d. h. der Verbindung zwischen Politikern und der Glücksspielbranche im Rahmen von Änderungen des } \\
\text { Glücksspielgesetzes, und zwei Ergänzungen aus den Anträgen der Oppositionsparteien stattzugeben. Der Antrag } \\
\text { der PO legt den Untersuchungszeitraum auf die Regierungszeit der Demokratischen Linksallianz (Sojusz Lewicy } \\
\text { Demokratycznej-SLD) (2002) und von Recht und Gerechtigkeit (Prawo i Sprawiedliwość-PiS) bis zum Bekannt- } \\
\text { werden der Affäre unter der Regierung der PO im Oktober } 2009 \text { fest. Die Anträge der Oppositionsparteien SLD } \\
\text { und PiS, die einen Untersuchungszeitraum von November } 2007 \text { bis Oktober } 2009 \text { (Regierungszeit der Koalition aus } \\
\text { PO und der Polnischen Bauernpartei/Polskie Stronnictwo Demokratyczne - PSL) vorsahen, wurden abgelehnt. }\end{array}$ \\
\hline 03.11 .2009 & $\begin{array}{l}\text { Der Interimsvorsitzende des Aufsichtsrats des Polnischen Fernsehens (TVP), Bogusław Szwedo, tritt nach einem } \\
\text { Monat Amtszeit aus persönlichen Gründen zurück. Er kehrt damit als Mitglied in den Aufsichtsrat zurück. Szwedo } \\
\text { hatte bereits bei seinem Antritt angekündigt, seine neue Funktion nur wenige Monate ausüben zu wollen. Neuer } \\
\text { Interimschef des TVP wird bis zur Aufsichtsratswahl Tomasz Szatkowski, der seit wenigen Monaten Mitglied des } \\
\text { TVP-Aufsichtsrats ist. }\end{array}$ \\
\hline 05.11 .2009 & $\begin{array}{l}\text { Im Rahmen der polnisch-französischen Regierungskonsultationen werden gemeinsame Erklärungen zu den Bereichen } \\
\text { Sicherheit und Verteidigung, Landwirtschaft, Kultur sowie Energie, Umwelt und Klima unterzeichnet. Ministerprä- } \\
\text { sident Donald Tusk unterstreicht, dass beide Länder eine proatlantische Politik betreiben und sich für die Stärkung } \\
\text { der gemeinsamen Sicherheits- und Verteidigungspolitik der Europäischen Union einsetzen werden. Im Bereich des } \\
\text { Aufbaus der Nutzung von Kernenergie sei Frankreich für Polen vorrangiger Partner. }\end{array}$ \\
\hline 06.11 .2009 & $\begin{array}{l}\text { Sejmmarschall Bronisław Komorowski und Bundestagspräsident Norbert Lammert eröffnen im Sejm eine vom } \\
\text { Deutschen Bundestag vorbereitete Ausstellung aus Anlass des 20. Jahrestages des Falls der Berliner Mauer unter } \\
\text { dem Titel „Die Mauer fiel - die friedliche Revolution und die Überwindung der Teilung». }\end{array}$ \\
\hline 06.11 .2009 & $\begin{array}{l}\text { Nach einem Treffen mit Bundestagspräsident Norbert Lammert unterstreicht Sejmmarschall Bronisław Komorowski, } \\
\text { dass die polnische Seite ihren Standpunkt hinsichtlich der geplanten deutsch-russischen Gaspipeline nicht geändert } \\
\text { habe und beunruhigt sei, dass das Projekt die Verlegung der Pipeline durch die Ostsee vorsehe und Polen auf diese } \\
\text { Weise umgehe. Er wies darauf hin, dass auf Anregung Polens die Energiesolidarität zwischen den EU-Mitglieds- } \\
\text { ländern in den EU-Vertrag von Lissabon aufgenommen worden sei. Die herrschende Wirtschaftskrise könne } \\
\text { darüber hinaus zum Anlass genommen werden, noch einmal über eine kostengünstigere Variante des Landweges } \\
\text { für den Gastransfer nachzudenken. }\end{array}$ \\
\hline 08.11 .2009 & $\begin{array}{l}\text { Nach einer vom Institut für Öffentliche Angelegenheiten (Instytut Spraw Publicznych - ISP) veröffentlichten } \\
\text { Umfrage bewerten } 59 \% \text { der Polen die Situation in ihrem Land als besser als vor 1989. Derselben Meinung sind } \\
68 \% \text { der Tschechen, } 53 \% \text { der Slowaken und knapp } 28 \% \text { der Ungarn. }\end{array}$ \\
\hline 09.11 .2009 & $\begin{array}{l}\text { Ministerpräsident Donald Tusk und sein spanischer Amtskollege José Luis Rodríguez Zapatero unterzeichnen ein } \\
\text { Abkommen über den Verkauf von } \mathrm{CO}_{2} \text {-Emissionsrechten. Polen werde dadurch ca. eine halbe Milliarde Zloty } \\
\text { erhalten, die in eine umweltfreundlichere Ausstattung der polnischen Wirtschaft investiert würden, so Tusk. }\end{array}$ \\
\hline 09.11 .2009 & $\begin{array}{l}\text { Im Rahmen der Feierlichkeiten in Berlin zum 20. Jahrestag des Falls der Berliner Mauer dankt Bundeskanzlerin } \\
\text { Angela Merkel in ihrer Rede der polnischen Gewerkschaftsbewegung Solidarność, deren Erhebung gegen die } \\
\text { kommunistische Herrschaft eine Ermutigung für die anderen sozialistischen Länder gewesen sei. Der ehemalige } \\
\text { Solidarność-Führer und erste vom Volk gewählte Staatspräsident des demokratischen Polen, Lech Wałęsa, stößt im } \\
\text { Rahmen des Domino-Happenings den ersten Dominostein an, auf dem ein Bild des Papstes Johannes Paul II., des } \\
\text { Runden Tisches, des Denkmals der gefallenen Werftarbeiter in Danzig und eine Landkarte Polens zu sehen sind. }\end{array}$ \\
\hline 10.11 .2009 & $\begin{array}{l}\text { Nach Informationen des Statistischen Hauptamts (Główny Urząd Statystyczny - GUS) betrug der Durchschnitts- } \\
\text { lohn im 3. Quartal } 20093.114 \text { Zloty und stieg damit im Vergleich zum 3. Quartal } 2008 \text { um 4,8 \%. Im Vergleich } \\
\text { zum 2. Quartal } 2009 \text { stieg der Durchschnittslohn um } 1 \% \text { und war damit geringer als die Inflationsrate. }\end{array}$ \\
\hline 11.11.2009 & $\begin{array}{l}\text { In Warschau finden die Hauptfeierlichkeiten des Unabhängigkeitstags (11. November 1918) statt, an denen u. a. } \\
\text { Staatspräsident Lech Kaczyński, Ministerpräsident Donald Tusk, Außenminister Radosław Sikorski und Ver- } \\
\text { teidigungsminister Bogdan Klich teilnehmen. Staatspräsident Kaczyński hebt hervor, dass Polen eine patriotische } \\
\text { Haltung entwickeln müsse, die an der Zukunft orientiert sei. Patriotismus sei heute der Kampf um Polens Platz in } \\
\text { Europa, um seine Sicherheit, wozu auch die energiepolitische Sicherheit gehöre, seine wirtschaftliche Entwicklung, } \\
\text { die Modernisierung des Landes und die Bewahrung von Werten. }\end{array}$ \\
\hline
\end{tabular}




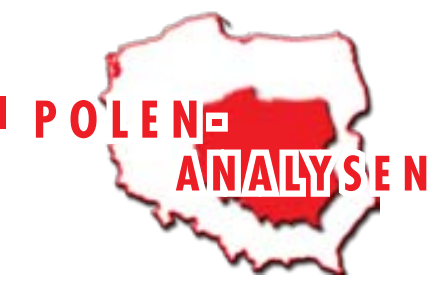

\begin{tabular}{|c|c|}
\hline 12.11 .2009 & $\begin{array}{l}\text { Während der diesjährigen Feierlichkeiten aus Anlass des 20. Jahrestags der deutsch-polnischen Versöhnungsmesse } \\
\text { in Kreisau (Krzyżowa), in der seinerzeit Ministerpräsident Tadeusz Mazowiecki und Bundeskanzler Helmut Kohl } \\
\text { den christlichen Versöhnungsgruß ausgetauscht hatten, weist Mazowiecki darauf hin, dass die deutsch-polnische } \\
\text { Versöhnung bereits eine Tatsache sei und nun an der polnisch-russischen Versöhnung gearbeitet werden müsse. Die } \\
\text { Rolle der deutschen Vertriebenenorganisationen und Erika Steinbachs als deren Vorsitzender sollte in Polen nicht } \\
\text { überinterpretiert werden, um die Position der nach seiner Einschätzung in Deutschland weitgehend unbekannten } \\
\text { öffentlichen Person nicht zu stärken. }\end{array}$ \\
\hline 13.11 .2009 & $\begin{array}{l}\text { Der Sprecher der Demokratischen Linksallianz (Sojusz Lewicy Demokratycznej - SLD), Tomasz Kalita, teilt mit, } \\
\text { dass seine Fraktion einen Antrag auf eine Stellungnahme der Regierung zur zweijährigen Amtszeit der Koalition aus } \\
\text { Bürgerplattform (Platforma Obywatelska - PO) und Polnischer Bauernpartei (Polskie Stronnictwo Ludowe - PSL) } \\
\text { im Sejm eingereicht hat. Die Bürger hätten das Recht auf umfassende Informationen zur Umsetzung der von der } \\
\text { Regierung angekündigten Vorhaben und zum Umgang mit der Wirtschaftskrise, heißt es in der Begründung. }\end{array}$ \\
\hline 15.11 .2009 & $\begin{array}{l}\text { Nach einer Internet-Umfrage des Business Centre Club (BCC) ist ein Fünftel der dem BCC angehörenden Manager } \\
\text { und Firmeninhaber in Polen der Meinung, dass Ministerpräsident Donald Tusk ratlos und } 31 \% \text {, dass er eher ratlos } \\
\text { der Bekämpfung der Rezession gegenüber stünde. } 35 \% \text { der Respondenten urteilen, dass die Regierung den Markt- } \\
\text { bedürfnissen eher angemessen, fast dieselbe Anzahl, dass sie eher nicht angemessen und } 26 \% \text {, dass die Regierung } \\
\text { auf keinen Fall angemessen das polnische Unternehmertum schützt. }\end{array}$ \\
\hline 15.11. 2009 & $\begin{array}{l}\text { Der ehemalige Koordinator der Sicherheitsdienste (Służby Specjalne) in der Regierung von Jarosław Kaczyński, } \\
\text { Zbigniew Wassermann, kritisiert, dass die Regierung von Donald Tusk in den zwei Jahren ihrer Amtszeit kein Kon- } \\
\text { zept für die Aufsicht über die Sicherheitsdienste entwickelt habe. Es sei ein Fehler gewesen, keinen ministerialen } \\
\text { Koordinator für diesen Bereich berufen zu haben. Derzeit sei zu beobachten, dass die Sicherheitsdienste eine } \\
\text { zunehmende Eigendynamik entwickelten und nicht kontrolliert würden. }\end{array}$ \\
\hline 16.11.2009 & $\begin{array}{l}\text { Nach der neuesten Umfrage von GfK Polonia im Auftrag der Tageszeitung »Rzeczpospolita« zur Regierungspartei } \\
\text { Bürgerplattform (Platforma Obywatelska - PO) aus Anlass der zweijährigen Amtszeit der Regierung von Minister- } \\
\text { präsident Donald Tusk sind } 47 \% \text { der Auffassung, dass die PO kein gutes Führungspersonal aufweise; } 43 \% \text { sind } \\
\text { gegenteiliger Meinung. Einen Mangel an Experten in der PO sehen } 51 \% \text {. Nach Einschätzung von } 54 \% \text { hilft die PO } \\
\text { nicht den Unternehmen in der Wirtschaftskrise. } 66 \% \text { meinen, dass die PO ihre Wahlversprechen nicht umsetzt. }\end{array}$ \\
\hline
\end{tabular}




\title{
Call for Applications - $5^{\text {th }}$ Changing Europe Summer School
}

\author{
"Informal Networks, Clientelism and Corruption in Politics, State Administration, Business \\ and Society. Case studies from Central and Eastern Europe" \\ Institute of Sociology, Czech Academy of Sciences \\ Prague $1-7$ August 2010
}

organized by the Research Centre for East European Studies (University of Bremen) at the Institute of Sociology, Czech Academy of Sciences with funding from the Volkswagen Foundation

The topic: Informal networks, clientelism and corruption are often seen as legacies of socialism with a strong impact on post-socialist transformations, on the other hand they describe universal phenomena, which can be found in all kinds of societies. Although these phenomena are frequently argued to impede development, some authors also highlight their potential to make things work by offering a way around red tape and authoritarian pressures.

In order to obtain a better understanding of these phenomena, including their different forms, modes of function, causes and consequences in various societal contexts, the Changing Europe Summer School 2010 wants to offer a forum for empirical research on informal networks, clientelism and corruption with a regional focus on Central and Eastern Europe. Central and Eastern Europe is defined as the formerly socialist part of Europe including all countries of the CIS. Comparative approaches (across countries and across time) are especially encouraged.

The Summer School: Each year the Changing Europe Summer School brings together 20 to 30 young academics (i.e. mainly doctoral students from disciplines like political science, sociology, economics, social anthropology, law, geography and history) working on issues related to countries in Central and Eastern Europe. Participation in the Summer School gives them a chance to present and discuss their research projects and to become better integrated into the academic community.

The core of the Summer School consists of the presentation of doctoral research projects and their discussion with senior researchers of international repute in their respective fields. In addition, there will be several sessions with experts on funding, access to information, publication strategies and policy consulting. The sessions will be framed by lectures and excursions as well as other activities designed to give participants the opportunity to socialize and establish contacts. Selected contributions to the Summer School will be published in an edited volume. The participants will be invited to join our alumni network.

Paper proposals: Paper proposals must be based on original doctoral research projects and may not exceed 1000 words. They must be drafted in English and must connect an empirical question with a theoretical approach and concept in order to be accepted. An international review panel will assess the papers for the conference in an anonymous review process (for more information about the reviewers, see www.changing-europe.de). The deadline for receipt of paper proposals is 10 January 2010. Please submit your proposal according to the guidelines at www.changing-europe.de.

Costs: Funding by the Volkswagen Foundation covers accommodation and participation fees. Participants will have to cover their travel costs themselves.

Location: Institute of Sociology, Czech Academy of Sciences, Prague

Information: More information about the Changing Europe Summer Schools is available at www.changing-europe.de 


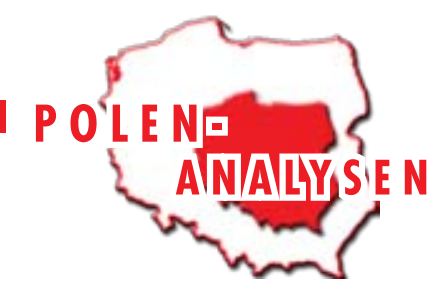

Über die Polen-Analysen

Die Polen-Analysen erscheinen zweimal monatlich als E-Mail-Dienst. Sie werden gemeinsam vom Deutschen PolenInstitut Darmstadt, der Bremer Forschungsstelle Osteuropa und der Deutschen Gesellschaft für Osteuropakunde herausgegeben.

Ein Archiv der Polen-Analysen finden Sie im Internet unter www.laender-analysen.de/polen

Kostenloses Abonnement unter http://www.deutsches-polen-institut.de/Newsletter/subscribe.php

\section{Deutsches Polen-Institut Darmstadt}

Das Deutsche Polen-Institut Darmstadt (DPI) ist ein Forschungs-, Informations-, und Veranstaltungszentrum für polnische Kultur, Geschichte, Politik, Gesellschaft und die deutsch-polnischen Beziehungen, die sich im Kontext der europäischen Integration entwickeln. Das seit März 1980 aktive und bis 1997 von Gründungsdirektor Karl Dedecius geleitete Institut ist eine Gemeinschaftsgründung der Stadt Darmstadt, der Länder Hessen und Rheinland-Pfalz sowie des Bundes. Seit 1987 ist die Trägerschaft auf die Kultusminister der Länder ausgedehnt. Einen wesentlichen Beitrag zur Verwirklichung der Institutsziele leisten private Stiftungen. Das DPI hat satzungsgemäß die Aufgabe, durch seine Arbeit zur Vertiefung der gegenseitigen Kenntnisse des kulturellen, geistigen und gesellschaftlichen Lebens von Polen und Deutschen beizutragen.

Ziel der Vermittlertätigkeit des DPI ist es, „die zu interessieren, auf die es politisch, wirtschaftlich, gesellschaftlich und kulturell im deutsch-polnischen Verhältnis ankommt" (Leitlinien 1997). Es geht um die Entscheider und Multiplikatoren in Politik, Kultur, Bildung, Verwaltung, Medien und Wirtschaft und, wesentlich stärker ausgeprägt als bisher, um das Hineinwirken in Wissenschaft, Forschung und Bildung.

Derzeit bemüht sich das DPI in Kooperation mit den verstreuten Orten wissenschaftlicher Polen-Kompetenz an deutschen Hochschulen und Forschungsinstituten verstärkt darum, ausgehend von einer Bestandsaufnahme deutscher Polen-Forschung Ort wissenschaftlicher Forschung und verbindendes, vernetzendes und kooperierendes Zentrum zu werden. Ausgangspunkt der Neuausrichtung ist die kaum mehr kontrollierbare Dynamik des Rückbaus der Ressourcen der wissenschaftlichen Polen-Kompetenz in den unterschiedlichen Disziplinen. Mit der über 55.000 Bände zählenden multidisziplinären Fachbibliothek für Polen mit einer einzigartigen Sammlung polnischer Literatur in der Originalsprache und in deutscher Übersetzung ist das DPI bereits ein geschätzter Ort der Recherche und des wissenschaftlichen Arbeitens. (www.deutsches-polen-institut.de)

\section{Forschungsstelle Osteuropa an der Universität Bremen}

1982 gegründet, widmet sich die Forschungsstelle Osteuropa an der Universität Bremen kulturellen und gesellschaftlichen Entwicklungen der Länder Ost- und Ostmitteleuropas in Zeitgeschichte und Gegenwart. Die Forschungsstelle besitzt in ihrem Archiv eine einzigartige Sammlung alternativer Kulturgüter und unabhängiger Texte aus den ehemaligen sozialistischen Ländern. Darunter befindet sich auch eine umfangreiche Sammlung des "Zweiten Umlaufs«, die das Schrifttum und Dokumente unabhängiger Initiativen und gesellschaftlicher Gruppen in Polen aus der Zeit von 1976 bis zum Umbruch umfasst. Neben ausführlicher individueller Forschung zu Dissens und Gesellschaft im Sozialismus, leitet die Forschungsstelle seit Januar 2007 ein gemeinsames Projekt mit einem Verbund von internationalen Forschungsinstituten zum Thema "Das andere Osteuropa - die 1960er bis 1980er Jahre, Dissens in Politik und Gesellschaft, Alternativen in der Kultur. Beiträge zu einer vergleichenden Zeitgeschichte«, welches von der VolkswagenStiftung finanziert wird.

Im Bereich der post-sozialistischen Gesellschaften sind in den letzten Jahren umfangreiche Forschungsprojekte durchgeführt worden, deren Schwerpunkte auf politischen Entscheidungsprozessen, Wirtschaftskultur und der EU-Osterweiterung lagen. Eine der Hauptaufgaben der Forschungsstelle ist die Information der interessierten Öffentlichkeit. Dazu gehören unter anderem regelmäßige E-Mail-Informationsdienste mit fast 15.000 Abonnenten in Politik, Wirtschaft und den Medien.

Mit ihrer in Deutschland einzigartigen Sammlung von Publikationen zu Osteuropa ist die Forschungsstelle eine Anlaufstelle sowohl für Wissenschaftler als auch für die interessierte Öffentlichkeit. In der Bibliothek sind derzeit neben anderen breit angelegten Beständen allein aus Polen ca. 300 laufende Periodika zugänglich. Die Bestände werden in Datenbanken systematisch erfasst. (www.forschungsstelle.uni-bremen.de)

Die Meinungen, die in den Polen-Analysen geäußert werden, geben ausschließlich die Auffassung der Autoren wieder. Abdruck und sonstige publizistische Nutzung sind nach Rücksprache mit der Redaktion gestattet. Redaktion: Prof. Dr. Dieter Bingen (Darmstadt), Silke Plate, M.A. (Bremen) Technische Gestaltung: Matthias Neumann

Polen-Analysen-Layout: Cengiz Kibaroglu, Matthias Neumann

ISSN 1863-9712 (C) 2009 by Deutsches Polen-Institut Darmstadt und Forschungsstelle Osteuropa, Bremen Kontakt: Dr. Andrzej Kaluza, Presse- und Öffentlichkeitsarbeit, Deutsches Polen-Institut, Mathildenhöhweg 2, 